\title{
A New System of Generalized Mixed Quasivariational Inclusions with Relaxed Cocoercive Operators and Applications
}

\author{
Zhongping Wan, ${ }^{1}$ Jia-Wei Chen, ${ }^{1,2}$ Hai Sun, ${ }^{2}$ and Liuyang Yuan' ${ }^{1}$ \\ ${ }^{1}$ School of Mathematics and Statistics, Wuhan University, Wuhan, Hubei 430072, China \\ ${ }^{2}$ School of Mathematics and Information, China West Normal University, Nanchong, \\ Sichuan 637002, China \\ Correspondence should be addressed to Zhongping Wan, mathwanzhp@whu.edu.cn
}

Received 21 April 2011; Accepted 12 June 2011

Academic Editor: Yongkun Li

Copyright (C) 2011 Zhongping Wan et al. This is an open access article distributed under the Creative Commons Attribution License, which permits unrestricted use, distribution, and reproduction in any medium, provided the original work is properly cited.

\begin{abstract}
A new system of generalized mixed quasivariational inclusions (for short, SGMQVI) with relaxed cocoercive operators, which develop some preexisting variational inequalities, is introduced and investigated in Banach spaces. Next, the existence and uniqueness of solutions to the problem (SGMQVI) are established in real Banach spaces. From fixed point perspective, we propose some new iterative algorithms for solving the system of generalized mixed quasivariational inclusion problem (SGMQVI). Moreover, strong convergence theorems of these iterative sequences generated by the corresponding algorithms are proved under suitable conditions. As an application, the strong convergence theorem for a class of bilevel variational inequalities is derived in Hilbert space. The main results in this paper develop, improve, and unify some well-known results in the literature.
\end{abstract}

\section{Introduction}

Generalized mixed quasivariational inclusion problems, which are extensions of variational inequalities introduced by Stampacchia [1] in the early sixties, are among the most interesting and extensively investigated classes of mathematics problems and have many applications in the fields of optimization and control, abstract economics, electrical networks, game theory, auction, engineering science, and transportation equilibria (see, e.g., [2-5] and the references therein). For the past few decades, existence results and iterative algorithms for variational inequality and variational inclusion problems have been obtained (see, e.g., [6-14] and the references cited therein). Recently, some new problems, which are called to be the system of variational inequality and equilibrium problems, received many attentions. Ansari et al. [2] considered a system of vector variational inequalities and obtained its existence results. 
In [3], Pang stated that the traffic equilibrium problem, the spatial equilibrium problem, the Nash equilibrium, and the general equilibrium programming problem can be modeled as a system of variational inequalities. Verma [15] and J. K. Kim and D. S. Kim [16] investigated a system of nonlinear variational inequalities. Cho et al. [17] introduced and studied a new system of nonlinear variational inequalities in Hilbert spaces and obtained the existence and uniqueness properties of solutions for the system of nonlinear variational inequalities. In [18], Peng and $\mathrm{Zhu}$ introduced a new system of generalized mixed quasivariational inclusions involving $(H, \eta)$-monotone operators. Very recently, Qin et al. [19] studied the approximation of solutions to a system of variational inclusions in Banach spaces and established a strong convergence theorem in uniformly convex and 2 uniformly smooth Banach spaces. In [20], Kamraksa and Wangkeeree gave a general iterative method for a general system of variational inclusions and proved a strong convergence theorem in strictly convex and 2 uniformly smooth Banach spaces. Further, Wangkeeree and Kamraksa [21] introduced an iterative algorithm for finding a common element of the set of solutions of a mixed equilibrium problem, the set of fixed points of an infinite family of nonexpansive mappings and the set of solutions of a general system of variational inequalities and then obtained the strong convergence of the iterative in Hilbert spaces. Petrot [22] applied the resolvent operator technique to find the common solutions for a generalized system of relaxed cocoercive mixed variational inequality problems and fixed point problems for Lipschitz mappings in Hilbert spaces. Zhao et al. [23] obtained some existence results for a system of variational inequalities by Brouwer's fixed point theory and proved the convergence of an iterative algorithm in finite Euclidean spaces. Chen and Wan [24] also proved the existence of solutions and convergence analysis for a system of quasivariational inclusions in Banach spaces, proposed some iterative methods for finding the common element of the solutions set for the system of quasivariational inclusions and the fixed point set for Lipschitz mapping, and obtained the convergent rates of corresponding iterative sequences. On the other hand, various bilevel programming problems, bilevel decision problems, and mathematical program problems with equilibrium (variational inequalities) constraints have been wildly investigated (see, e.g., $[25,26])$. To the best of our knowledge, there is few results concerning the algorithms and convergence analysis of solutions to bilevel variational inequalities in Hilbert spaces.

The aim of this paper is to introduce and study a new system of generalized mixed quasivariational inclusion problem (SGMQVI) in uniformly smooth Banach spaces which includes some previous variational inequalities as special cases. Furthermore, the existence and uniqueness theorems of solutions for the problem (SGMQVI) are established by using resolvent techniques. Thirdly, we also propose some new iterative algorithms for solving the problem (SGMQVI). Strong convergence of the iterative sequences generated by the corresponding iterative algorithms are proved under suitable conditions. As an application, we study the properties for the lower-level variational inequalities of a class of bilevel variational inequalities (for short, (BVI)) in Hilbert spaces and then suggest a reasonable iterative algorithm for (BVI). Finally, the strong convergence theorem for (BVI) are derived under appropriate assumptions. The results presented in this paper improve, develop, and extend the results of $[8,23,24,27]$.

\section{Preliminaries}

Throughout this paper, let $E$ be a real $q$-uniformly Banach space with its dual $E^{*}, q>1$, denote the duality between $E$ and $E^{*}$ by $\langle\cdot, \cdot\rangle$ and the norm of $E$ by $\|\cdot\|$, and let $T: E \rightarrow E$ be 
a nonlinear mapping. If $\left\{x_{n}\right\}$ is a sequence in $E$, we denote strong convergence of $\left\{x_{n}\right\}$ to $x \in E$ by $x_{n} \rightarrow x$. A Banach space $E$ is called smooth if $\lim _{t \rightarrow 0}(\|x+t y\|-\|x\|) / t$ exists for all $x, y \in E$ with $\|x\|=\|y\|=1$. It is called uniformly smooth if the limit is attained uniformly for $\|x\|=\|y\|=1$. The function

$$
\rho_{E}(t)=\sup \left\{\frac{\|x+y\|+\|x-y\|}{2}-1:\|x\|=1,\|y\| \leq t\right\}
$$

is called the modulus of smoothness of $E$. $E$ is called $q$-uniformly smooth if there exists a constant $c_{q}>0$ such that $\rho_{E}(t) \leq c_{q} t^{q}$.

Example 2.1 (see [4]). All Hilbert spaces, $L^{p}\left(\right.$ or $\left.l^{p}\right)$, and the Sobolev spaces $W_{m}^{p},(p \geq 2)$ are 2-uniformly smooth, while $L^{p}\left(\right.$ or $\left.l^{p}\right)$ and $W_{m}^{p}$ spaces $(1<p \leq 2)$ are $p$-uniformly smooth.

The generalized duality mapping $J_{q}: E \rightarrow 2^{E^{*}}$ defined as follows:

$$
J_{q}(x)=\left\{f^{*} \in E^{*}:\left\langle f^{*}, x\right\rangle=\left\|f^{*}\right\|\|x\|=\|x\|^{q},\left\|f^{*}\right\|=\|x\|^{q-1}\right\},
$$

for all $x \in E$. As we know that $J=J_{2}$ is the usual normalized duality mapping, and $J_{q}(x)=\|x\|^{q-2} J(x)$ for $x \neq 0, J_{q}(t x)=t^{q-1} J_{q}(x)$, and $J_{q}(-x)=-J_{q}(x)$ for all $x \in E$ and $t \in[0,+\infty)$, and $J_{q}$ is single-valued if $E$ is smooth (see, e.g., [28]). If $E$ is a Hilbert space, then $J=I$, where $I$ is the identity operator. Let $g_{j}: E \rightarrow E$, let $A_{j}: E \times E \rightarrow E$ be single-valued mappings, and let $M_{j}: E \rightarrow 2^{E}$ be set-valued mappings for all $j \in\{1,2, \ldots, n\}$. We consider the system of generalized mixed quasivariational inclusions problem (for short, (SGMQVI)) as follows: find $\left(x_{1}^{*}, x_{2}^{*}, \ldots, x_{n-1}^{*}, x_{n}^{*}\right) \in E^{n}$ such that

$$
\begin{gathered}
0 \in x_{1}^{*}-g_{1}\left(x_{2}^{*}\right)+\rho_{1}\left(A_{1}\left(x_{2}^{*}, x_{1}^{*}\right)+M_{1}\left(x_{1}^{*}\right)\right), \\
0 \in x_{2}^{*}-g_{2}\left(x_{3}^{*}\right)+\rho_{2}\left(A_{2}\left(x_{3}^{*}, x_{2}^{*}\right)+M_{2}\left(x_{2}^{*}\right)\right), \\
\vdots \\
0 \in x_{n-1}^{*}-g_{n-1}\left(x_{n}^{*}\right)+\rho_{n-1}\left(A_{n-1}\left(x_{n}^{*}, x_{n-1}^{*}\right)+M_{n-1}\left(x_{n-1}^{*}\right)\right), \\
0 \in x_{n}^{*}-g_{n}\left(x_{1}^{*}\right)+\rho_{n}\left(A_{n}\left(x_{1}^{*}, x_{n}^{*}\right)+M_{n}\left(x_{n}^{*}\right)\right),
\end{gathered}
$$

where $\rho_{i}(i=1,2, \ldots, n)$ are positive constants. Denote the set of solutions to (SGMQVI) by $\Xi$. 
Special cases are as follows:

(I) If $g_{j}(x)=x$ and $A_{j}(x, y)=T_{j}(x)+S_{j}(x)$ for all $x, y \in E$ and $j=1,2, \ldots, n$, where $T_{j}, S_{j}: E \rightarrow E$ are single-valued mappings, then the problem (SGMQVI) is equivalent to find $\left(x_{1}^{*}, x_{2}^{*}, \ldots, x_{n-1}^{*}, x_{n}^{*}\right) \in E^{n}$ such that

$$
\begin{gathered}
0 \in x_{1}^{*}-x_{2}^{*}+\rho_{1}\left(T_{1}\left(x_{2}^{*}\right)+S_{1}\left(x_{2}^{*}\right)+M_{1}\left(x_{1}^{*}\right)\right), \\
0 \in x_{2}^{*}-x_{3}^{*}+\rho_{2}\left(T_{2}\left(x_{3}^{*}\right)+S_{2}\left(x_{3}^{*}\right)+M_{2}\left(x_{2}^{*}\right)\right), \\
\vdots \\
0 \in x_{n-1}^{*}-x_{n}^{*}+\rho_{n-1}\left(T_{n-1}\left(x_{n}^{*}\right)+S_{n-1}\left(x_{n}^{*}\right)+M_{n-1}\left(x_{n-1}^{*}\right)\right), \\
0 \in x_{n}^{*}-x_{1}^{*}+\rho_{n}\left(T_{n}\left(x_{1}^{*}\right)+S_{n}\left(x_{1}^{*}\right)+M_{n}\left(x_{n}^{*}\right)\right),
\end{gathered}
$$

where $\rho_{i}(i=1,2, \ldots, n)$ are positive constants, which is called the system of generalized nonlinear mixed variational inclusions problem [8].

(II) If $n=2, A_{1}=A_{2}=A, E=H$ is a Hilbert space, $g_{1}(x)=g_{2}(x)=x$ and $M_{1}(x)=M_{2}(x)=\partial \phi(x)$ for all $x \in E$, where $\phi: E \rightarrow R \cup\{+\infty\}$ is a proper, convex, and lower semicontinuous functional, and $\partial \phi$ denotes the subdifferential operator of $\phi$, then the problem (SGMQVI) is equivalent to find $\left(x^{*}, y^{*}\right) \in E \times E$ such that

$$
\begin{array}{ll}
\left\langle\rho_{1} A\left(y^{*}, x^{*}\right)+x^{*}-y^{*}, x-x^{*}\right\rangle+\phi(x)-\phi\left(x^{*}\right) \geq 0, & \forall x \in E, \\
\left\langle\rho_{2} A\left(x^{*}, y^{*}\right)+y^{*}-x^{*}, x-y^{*}\right\rangle+\phi(x)-\phi\left(y^{*}\right) \geq 0, & \forall x \in E,
\end{array}
$$

where $\rho_{i}(i=1,2)$ are positive constants, which is called the generalized system of relaxed cocoercive mixed variational inequality problem [29].

(III) If $n=2, E=H$ is a Hilbert space, and $K$ is a closed convex subset of $E$, and $\phi(x)=\delta_{K}(x)$ for all $x \in K$, where $\delta_{K}$ is the indicator function of $K$ defined by

$$
\phi(x)=\delta_{K}(x)= \begin{cases}0, & \text { if } x \in K \\ +\infty, & \text { otherwise }\end{cases}
$$

then the problem (SGMQVI) is equivalent to find $\left(x^{*}, y^{*}\right) \in K \times K$ such that

$$
\begin{array}{ll}
\left\langle\rho_{1} A_{1}\left(y^{*}, x^{*}\right)+x^{*}-g_{1}\left(y^{*}\right),\right. & \left.g_{1}(x)-x^{*}\right\rangle \geq 0, \quad \forall x \in K, \\
\left\langle\rho_{2} A_{2}\left(x^{*}, y^{*}\right)+y^{*}-g_{2}\left(x^{*}\right),\right. & \left.g_{2}(x)-y^{*}\right\rangle \geq 0, \quad \forall x \in K,
\end{array}
$$

where $\rho_{i}(i=1,2)$ are positive constants, which is called the system of general variational inequalities problem [27]. 
(IV) If $n=2, A_{1}=A_{2}=A, E=H$ is a Hilbert space, and $K$ is a closed convex subset of $E, g_{1}(y)=g_{2}(y)=y$, and $\phi(x)=\delta_{K}(x)$ for all $x \in K, y \in E$, where $\delta_{K}$ is the indicator function of $K$ defined by

$$
\phi(x)=\delta_{K}(x)= \begin{cases}0, & \text { if } x \in K \\ +\infty, & \text { otherwise }\end{cases}
$$

then the problem (SGMQVI) is equivalent to find $\left(x^{*}, y^{*}\right) \in K \times K$ such that

$$
\begin{array}{ll}
\left\langle\rho_{1} A\left(y^{*}, x^{*}\right)+x^{*}-y^{*}, x-x^{*}\right\rangle \geq 0, & \forall x \in K, \\
\left\langle\rho_{2} A\left(x^{*}, y^{*}\right)+y^{*}-x^{*}, x-y^{*}\right\rangle \geq 0, & \forall x \in K,
\end{array}
$$

where $\rho_{i}(i=1,2)$ are positive constants, which is called the generalized system of relaxed cocoercive variational inequality problem [30].

(V) If for each $i \in\{1,2\}, z \in E, A_{i}(x, z)=\Psi_{i}(x)$, and $g_{i}(x)=x$ for all $x \in E$, where $\Psi_{i}: E \rightarrow E$, then the problem (SGMQVI) is equivalent to find $\left(x^{*}, y^{*}\right) \in E \times E$ such that

$$
\begin{aligned}
& 0 \in x^{*}-y^{*}+\rho_{1}\left(\Psi_{1}\left(y^{*}\right)+M_{1}\left(x^{*}\right)\right), \\
& 0 \in y^{*}-x^{*}+\rho_{2}\left(\Psi_{2}\left(x^{*}\right)+M_{2}\left(y^{*}\right)\right),
\end{aligned}
$$

where $\rho_{i}(i=1,2)$ are positive constants, which is called the system of quasivariational inclusion $[19,20]$.

(VI) If $n=2$, for each $i \in\{1,2\}, z \in E, A_{i}(x, z)=\Psi(x)$, and $g_{i}(x)=x$ for all $x \in E$, where $\Psi: E \rightarrow E$ and $M_{1}(x)=M_{2}(x)=M$, then the problem (SGMQVI) is equivalent to find $\left(x^{*}, y^{*}\right) \in E \times E$ such that

$$
\begin{aligned}
& 0 \in x^{*}-y^{*}+\rho_{1}\left(\Psi\left(y^{*}\right)+M\left(x^{*}\right)\right), \\
& 0 \in y^{*}-x^{*}+\rho_{2}\left(\Psi\left(x^{*}\right)+M\left(y^{*}\right)\right),
\end{aligned}
$$

where $\rho_{i}(i=1,2)$ are positive constants, which is called the system of quasivariational inclusion [20].

We first recall some definitions and lemmas which are needed in our main results.

Definition 2.2. Let $M: \operatorname{dom}(M) \subset E \rightarrow 2^{E}$ be a set-valued mapping, where $\operatorname{dom}(M)$ is the effective domain of the mapping $M . M$ is said to be

(i) accretive if, for any $x, y \in \operatorname{dom}(M), u \in M(x)$ and $v \in M(y)$, there exists $j_{q}(x-y) \in$ $J_{q}(x-y)$ such that

$$
\left\langle u-v, j_{q}(x-y)\right\rangle \geq 0,
$$

(ii) $m$-accretive (maximal-accretive) if $M$ is accretive and $(I+\rho M) \operatorname{dom}(M)=E$ holds for every $\rho>0$, where $I$ is the identity operator on $E$. 
Remark 2.3. If $E$ is a Hilbert space, then accretive operator and $m$-accretive operator are reduced to monotone operator and maximal monotone operator, respectively.

Definition 2.4 (see $[24,31]$ ). Let $T: E \rightarrow E$ be a single-valued mapping. $T$ is said to be

(i) $\gamma$-Lipschitz continuous mapping if there exists a constant $\gamma>0$ such that

$$
\|T x-T y\| \leq r\|x-y\|, \quad \forall x, y \in E,
$$

(ii) $(a, b)$-relaxed cocoercive if there exist two constants $a \geq 0$ and $b>0$ such that

$$
\left\langle T(x)-T(\tilde{x}), J_{q}(x-\tilde{x})\right\rangle \geq(-a)\|T(x)-T(\tilde{x})\|^{q}+b\|x-\tilde{x}\|^{q}, \quad \forall x, \tilde{x} \in E .
$$

Remark 2.5 (see [24]). (1) If $\gamma=1$, then a $\gamma$-Lipschitz continuous mapping reduces to a nonexpansive mapping.

(2) If $\gamma \in(0,1)$, then a $\gamma$-Lipschitz continuous mapping reduces to a contractive mapping.

(3) It is easy to see that the identity operator $I: E \rightarrow E$ is $(0,1)$ relaxed cocoercive, where $I(x)=x$ for all $x \in E$.

Definition 2.6 (see [24]). Let $A: E \times E \rightarrow E$ be a mapping. $A$ is said to be

(i) $\tau$-Lipschitz continuous in the first variable if there exists a constant $\tau>0$ such that, for $x, \tilde{x} \in E$,

$$
\|A(x, y)-A(\tilde{x}, \tilde{y})\| \leq \tau\|x-\tilde{x}\|, \quad \forall y, \tilde{y} \in E
$$

(ii) $\alpha$-strongly accretive if there exists a constant $\alpha>0$ such that

$$
\left\langle A(x, y)-A(\tilde{x}, \tilde{y}), J_{q}(x-\tilde{x})\right\rangle \geq \alpha\|x-\tilde{x}\|^{q}, \quad \forall(x, y),(\tilde{x}, \tilde{y}) \in E \times E,
$$

or equivalently,

$$
\langle A(x, y)-A(\tilde{x}, \tilde{y}), J(x-\tilde{x})\rangle \geq \alpha\|x-\tilde{x}\|, \quad \forall(x, y),(\tilde{x}, \tilde{y}) \in E \times E,
$$

(iii) $(\mu, v)$ relaxed cocoercive if there exist two constants $\mu \geq 0$ and $v>0$ such that

$$
\begin{array}{r}
\left\langle A(x, y)-A(\tilde{x}, \tilde{y}), J_{q}(x-\tilde{x})\right\rangle \geq(-\mu)\|A(x, y)-A(\tilde{x}, \tilde{y})\|^{q}+v\|x-\tilde{x}\|^{q}, \\
\forall(x, y),(\tilde{x}, \tilde{y}) \in E \times E .
\end{array}
$$

Remark 2.7. (1) Every $\alpha$-strongly accretive mapping is a $(\mu, \alpha)$ relaxed cocoercive for any positive constant $\mu$. But the converse is not true in general. 
(2) The conception of the cocoercivity is applied in several directions, especially for solving variational inequality problems by using the auxiliary problem principle and projection methods [14]. Several classes of relaxed cocoercive variational inequalities have been investigated in $[5,22,28,30]$.

Definition 2.8 (see $[9,32]$ ). Let the set-valued mapping $M: \operatorname{dom}(M) \subset E \rightarrow 2^{E}$ be $m$ accretive. For any positive number $\rho>0$, the mapping $R_{\rho}^{M}: E \rightarrow \operatorname{dom}(M)$ defined by

$$
R_{\rho}^{M}(x)=(I+\rho M)^{-1}(x), \quad x \in E,
$$

is called the resolvent operator associated with $M$ and $\rho$, where $I$ is the identity operator on $E$.

Remark 2.9. Let $C \subset E$ be a nonempty closed convex set. If $E$ is a Hilbert space and $M=\partial \phi$, the subdifferential of the indicator function $\phi$, that is,

$$
\phi(x)=\delta_{C}(x)= \begin{cases}0, & \text { if } x \in C \\ +\infty, & \text { otherwise }\end{cases}
$$

then $R_{(\rho, M)}=P_{C}$, the metric projection operator from $E$ onto $C$.

Lemma 2.10 (see $[9,32]$ ). Let the set-valued mapping $M: \operatorname{dom}(M) \subset E \rightarrow 2^{E}$ be $m$-accretive. Then the resolvent operator $R_{\rho}^{M}$ is single-valued and nonexpansive for all $\rho>0$.

Lemma 2.11 (see [33]). Let $\left\{B_{n}\right\},\left\{C_{n}\right\}$, and $\left\{D_{n}\right\}$ be three nonnegative real sequences satisfying the following conditions:

$$
B_{n+1} \leq\left(1-\lambda_{n}\right) B_{n}+C_{n}+D_{n}, \quad \forall n \geq n_{0}
$$

for some $n_{0} \in N,\left\{\lambda_{n}\right\} \subset(0,1)$ with $\sum_{n=0}^{\infty} \lambda_{n}=\infty, C_{n}=0\left(\lambda_{n}\right)$ and $\sum_{n=0}^{\infty} D_{n}<+\infty$. Then $\lim _{n \rightarrow \infty} B_{n}=0$.

Lemma 2.12 (see [34]). Let E be a real $q$-uniformly Banach space. Then there exists a constant $c_{q}>0$ such that

$$
\|x+y\|^{q} \leq\|x\|^{q}+q\left\langle y, J_{q}(x)\right\rangle+c_{q}\|y\|^{q}, \quad \forall x, y \in E .
$$

\section{Existence Theorems}

In this section, we will investigate the existence and uniqueness of solutions for the problem (SGMQVI) in $q$-uniformly smooth Banach space under some suitable conditions. 
Theorem 3.1. Let $\left(x_{1}^{*}, x_{2}^{*}, \ldots, x_{n-1}^{*}, x_{n}^{*}\right) \in E^{n}, M_{i}: E \rightarrow 2^{E}(i=1,2, \ldots, n)$ be maximal accretive. Then $\left(x_{1}^{*}, x_{2}^{*}, \ldots, x_{n-1}^{*}, x_{n}^{*}\right)$ is a solution of the problem (SGMQVI) if and only if

$$
\begin{gathered}
x_{1}^{*}=R_{\rho_{1}}^{M_{1}}\left(g_{1}\left(x_{2}^{*}\right)-\rho_{1} A_{1}\left(x_{2}^{*}, x_{1}^{*}\right)\right), \\
x_{2}^{*}=R_{\rho_{2}}^{M_{2}}\left(g_{2}\left(x_{3}^{*}\right)-\rho_{2} A_{2}\left(x_{3}^{*}, x_{2}^{*}\right)\right), \\
\vdots \\
x_{n-1}^{*}=R_{\rho_{n-1}}^{M_{n-1}}\left(g_{n-1}\left(x_{n}^{*}\right)-\rho_{n-1} A_{n-1}\left(x_{n}^{*}, x_{n-1}^{*}\right)\right), \\
x_{n}^{*}=R_{\rho_{n}}^{M_{n}}\left(g_{n}\left(x_{1}^{*}\right)-\rho_{n} A_{n}\left(x_{1}^{*}, x_{n}^{*}\right)\right),
\end{gathered}
$$

where $\rho_{i}(i=1,2, \ldots, n)$ are positive constants.

Proof. Let $\left(x_{1}^{*}, x_{2}^{*}, \ldots, x_{n-1}^{*}, x_{n}^{*}\right) \in E^{n}$ be a solution of the problem (SGMQVI). Then, for any given positive constants $\rho_{i}(i=1,2, \ldots, n)$, the problem (SGMQVI) is equivalent to

$$
\begin{gathered}
g_{1}\left(x_{2}^{*}\right)-\rho_{1} A_{1}\left(x_{2}^{*}, x_{1}^{*}\right) \in x_{1}^{*}+\rho_{1} M_{1}\left(x_{1}^{*}\right), \\
g_{2}\left(x_{3}^{*}\right)-\rho_{2} A_{2}\left(x_{3}^{*}, x_{2}^{*}\right) \in x_{2}^{*}+\rho_{2} M_{2}\left(x_{2}^{*}\right), \\
\vdots \\
g_{n-1}\left(x_{n}^{*}\right)-\rho_{n-1} A_{n-1}\left(x_{n}^{*}, x_{n-1}^{*}\right) \in x_{n-1}^{*}+\rho_{n-1} M_{n-1}\left(x_{n-1}^{*}\right), \\
g_{n}\left(x_{1}^{*}\right)-\rho_{n} A_{n}\left(x_{1}^{*}, x_{n}^{*}\right) \in x_{n}^{*}+\rho_{n} M_{n}\left(x_{n}^{*}\right) .
\end{gathered}
$$

From Definition 2.8 and Lemma 2.10, it yields that (3.2) is equivalent to (3.1). This completes the proof.

Theorem 3.2. Let $E$ be a real q-uniformly smooth Banach space. Let $j \in\{1,2, \ldots, n\}, M_{j}: E \rightarrow 2^{E}$ be m-accretive mapping, Let $A_{j}: E \times E \rightarrow E$ be $\left(\mu_{j}, v_{j}\right)$-relaxed cocoercive and Lipschitz continuous in the first variable with constant $\tau_{j}$, and Let $g_{j}: E \rightarrow E$ be $\left(a_{j}, b_{j}\right)$-relaxed cocoercive and Lipschitz continuous with constant $\iota_{j}$. Then, for each $j \in\{1,2, \ldots, n\}, x \in E$, the mapping $R_{\rho_{j}}^{M_{j}}\left(g_{j}(x)-\right.$ $\left.\rho_{j} A_{j}(x, \cdot)\right): E \rightarrow E$ has at most one fixed point. If

$$
\min \left\{1+q a_{j} \iota_{j}^{q}+c_{q} \iota_{j}^{q}-q b_{j}, 1+q \rho_{j} \mu_{j} \tau_{j}^{q}+c_{q} \rho_{j}^{q} \tau_{j}^{q}-q \rho_{j} v_{j}\right\} \geq 0
$$

then the implicit function $y_{j}(x)$ determined by

$$
y_{j}(x)=R_{\rho_{j}}^{M_{j}}\left(g_{j}(x)-\rho_{j} A_{j}\left(x, y_{j}(x)\right)\right)
$$

is continuous on $E$. 
Proof. Let $x \in E$. We show by contradiction that $R_{\rho_{j}}^{M_{j}}\left(g_{j}(x)-\rho_{j} A_{j}(x, \cdot)\right): E \rightarrow E$ has at most one fixed point. Suppose to the contrary that $y, \tilde{y} \in E$ and $y \neq \tilde{y}$ such that

$$
\begin{aligned}
& y=R_{\rho_{j}}^{M_{j}}\left(g_{j}(x)-\rho_{j} A_{j}(x, y)\right), \\
& \tilde{y}=R_{\rho_{j}}^{M_{j}}\left(g_{j}(x)-\rho_{j} A_{j}(x, \tilde{y})\right) .
\end{aligned}
$$

Since $A_{j}$ is Lipschitz continuous in the first variable with constant $\tau_{j}$, then

$$
\begin{aligned}
\|y-\tilde{y}\| & =\left\|R_{\rho_{j}}^{M_{j}}\left(g_{j}(x)-\rho_{j} A_{j}(x, y)\right)-R_{\rho_{j}}^{M_{j}}\left(g_{j}(x)-\rho_{j} A_{j}(x, \tilde{y})\right)\right\| \\
& \leq\left\|g_{j}(x)-\rho_{j} A_{j}(x, y)-\left(g_{j}(x)-\rho_{j} A_{j}(x, \tilde{y})\right)\right\| \\
& =\rho_{j}\left\|A_{j}(x, y)-A_{j}(x, \tilde{y})\right\| \\
& \leq \rho_{j} \tau_{j}\|x-x\| \\
& =0
\end{aligned}
$$

which is a contradiction. Therefore, the mapping $R_{\rho_{j}}^{M_{j}}\left(g_{j}(x)-\rho_{j} A_{j}(x, \cdot)\right): E \rightarrow E$ has at most one fixed point.

Next, we show that the implicit function $y_{j}(x)$ is continuous on $E$. For any sequence, $\left\{x_{n}\right\} \subset E, x_{0} \in E, x_{n} \rightarrow x_{0}$ as $n \rightarrow \infty$. Since $A_{j}: E \times E \rightarrow E$ is $\left(\mu_{j}, v_{j}\right)$-relaxed cocoercive and Lipschitz continuous in the first variable with constant $\tau_{j}$ and $g_{j}: E \rightarrow E$ is $\left(a_{j}, b_{j}\right)$-relaxed cocoercive and Lipschitz continuous with constant $\iota_{j}$, one has

$$
\begin{aligned}
\widetilde{L}_{A_{j}}= & \left\|x_{n}-x_{0}-\rho_{j}\left[A_{j}\left(x_{n}, y\left(x_{n}\right)\right)-A_{j}\left(x_{0}, y\left(x_{0}\right)\right)\right]\right\|^{q} \\
\leq & \left\|x_{n}-x_{0}\right\|^{q}-q \rho_{j}\left\langle A_{j}\left(x_{n}, y\left(x_{n}\right)\right)-A_{j}\left(x_{0}, y\left(x_{0}\right)\right), J_{q}\left(x_{n}-x_{0}\right)\right\rangle \\
& +c_{q} \rho_{j}^{q}\left\|A_{j}\left(x_{n}, y\left(x_{n}\right)\right)-A_{j}\left(x_{0}, y\left(x_{0}\right)\right)\right\|^{q} \\
\leq & q \rho_{j}\left(\mu_{j}\left\|A_{j}\left(x_{n}, y\left(x_{n}\right)\right)-A_{j}\left(x_{0}, y\left(x_{0}\right)\right)\right\|^{q}-v_{j}\left\|x_{n}-x_{0}\right\|^{q}\right) \\
& +\left(1+c_{q} \rho_{j}^{q} \tau_{j}^{q}\right)\left\|x_{n}-x_{0}\right\|^{q} \\
\leq & q \rho_{j}\left(\mu_{j} \tau_{j}^{q}-v_{j}\right)\left\|x_{n}-x_{0}\right\|^{q}+\left(1+c_{q} \rho_{j}^{q} \tau_{j}^{q}\right)\left\|x_{n}-x_{0}\right\|^{q} \\
= & \left(1+q \rho_{j} \mu_{j} \tau_{j}^{q}+c_{q} \rho_{j}^{q} \tau_{j}^{q}-q \rho_{j} v_{j}\right)\left\|x_{n}-x_{0}\right\|^{q},
\end{aligned}
$$




$$
\begin{aligned}
\tilde{L}_{g_{j}} & =\left\|x_{n}-x_{0}-\left(g_{j}\left(x_{n}\right)-g_{j}\left(x_{0}\right)\right)\right\|^{q} \\
& \leq\left\|x_{n}-x_{0}\right\|^{q}-q\left\langle g_{j}\left(x_{n}\right)-g_{j}\left(x_{0}\right), J_{q}\left(x_{n}-x_{0}\right)\right\rangle+c_{q}\left\|g_{j}\left(x_{n}\right)-g_{j}\left(x_{0}\right)\right\|^{q} \\
& \leq\left(1+c_{q} \iota_{j}^{q}\right)\left\|x_{n}-x_{0}\right\|^{q}+q\left(a_{j}\left\|g_{j}\left(x_{n}\right)-g_{j}\left(x_{0}\right)\right\|^{q}-b_{j}\left\|x_{n}-x_{0}\right\|^{q}\right) \\
& \leq\left(1+c_{q} \iota_{j}^{q}\right)\left\|x_{n}-x_{0}\right\|^{q}+q\left(a_{j} \iota_{j}^{q}-b_{j}\right)\left\|x_{n}-x_{0}\right\|^{q} \\
& =\left(1+q a_{j} \iota_{j}^{q}+c_{q} q_{j}^{q}-q b_{j}\right)\left\|x_{n}-x_{0}\right\|^{q} .
\end{aligned}
$$

Therefore, from Lemma 2.10, we get

$$
\begin{aligned}
\left\|y_{j}\left(x_{n}\right)-y_{j}\left(x_{0}\right)\right\|= & \left\|R_{\rho_{j}}^{M_{j}}\left(g_{j}\left(x_{n}\right)-\rho_{j} A_{j}\left(x_{n}, y_{j}\left(x_{n}\right)\right)\right)-R_{\rho_{j}}^{M_{j}}\left(g_{j}\left(x_{0}\right)-\rho_{j} A_{j}\left(x_{0}, y_{j}\left(x_{0}\right)\right)\right)\right\| \\
\leq & \left\|g_{j}\left(x_{n}\right)-\rho_{j} A_{j}\left(x_{n}, y_{j}\left(x_{n}\right)\right)-\left(g_{j}\left(x_{0}\right)-\rho_{j} A_{j}\left(x_{0}, y_{j}\left(x_{0}\right)\right)\right)\right\| \\
= & \left\|\left(g_{j}\left(x_{n}\right)-g_{j}\left(x_{0}\right)\right)-\rho_{j}\left(A_{j}\left(x_{n}, y_{j}\left(x_{n}\right)\right)-A_{j}\left(x_{0}, y_{j}\left(x_{0}\right)\right)\right)\right\| \\
\leq & \left\|x_{n}-x_{0}-\left(g_{j}\left(x_{n}\right)-g_{j}\left(x_{0}\right)\right)\right\| \\
& +\left\|x_{n}-x_{0}-\rho_{j}\left(A_{j}\left(x_{n}, y_{j}\left(x_{n}\right)\right)-A_{j}\left(x_{0}, y_{j}\left(x_{0}\right)\right)\right)\right\| \\
\leq & \sqrt[q]{\widetilde{L}_{A_{j}}}+\sqrt[9]{\widetilde{L}_{g_{j}}} \\
\leq & \left(\sqrt[q]{1+q \rho_{j} \mu_{j} \tau_{j}^{q}+c_{q} \rho_{j}^{q} \tau_{j}^{q}-q \rho_{j} v_{j}}+\sqrt[q]{1+q a_{j} \iota_{j}^{q}+c_{q} \iota_{j}^{q}-q b_{j}}\right)\left\|x_{n}-x_{0}\right\| .
\end{aligned}
$$

From (3.3), it follows that the implicit function $y_{j}(x)$ is continuous on $E$. This completes the proof.

If $j=2$ and $g(x)=x$ for all $x \in E$, then Theorem 3.2 is reduced to the following result.

Corollary 3.3 (see [24]). Let E be a real q-uniformly smooth Banach space. Let $M_{2}: E \rightarrow 2^{E}$ be m-accretive mapping; Let $A_{2}: E \times E \rightarrow E$ be $\left(\mu_{2}, v_{2}\right)$-relaxed cocoercive and Lipschitz continuous in the first variable with constant $\tau_{2}$. Then, for each $x \in E$, the mapping $R_{\rho_{2}}^{M_{2}}\left(x-\rho_{2} A_{2}(x, \cdot)\right): E \rightarrow E$ has at most one fixed point. If

$$
1+q \rho_{2} \mu_{2} \tau_{2}^{q}+c_{q} \rho_{2}^{q} \tau_{2}^{q}-q \rho_{2} v_{2} \geq 0
$$

then the implicit function $y(x)$ determined by

$$
y(x)=R_{\rho_{2}}^{M_{2}}\left(x-\rho_{2} A_{2}\left(x, y_{2}(x)\right)\right)
$$

is continuous on $E$. 
Theorem 3.4. Let $E$ be a real $q$-uniformly smooth Banach space. Let $M_{j}: E \rightarrow 2^{E}$ be m-accretive mapping, Let $A_{j}: E \times E \rightarrow E$ be $\left(\mu_{j}, v_{j}\right)$-relaxed cocoercive and Lipschitz continuous in the first variable with constant $\tau_{j}$, and let $g_{j}: E \rightarrow E$ be $\left(a_{j}, b_{j}\right)$-relaxed cocoercive and Lipschitz continuous with constant $\iota_{j}$ for $j \in\{1,2, \ldots, n\}$. Assume that

$$
\begin{gathered}
\min \left\{1+q a_{j} \iota_{j}^{q}+c_{q} \iota_{j}^{q}-q b_{j}, 1+q \rho_{j} \mu_{j} \tau_{j}^{q}+c_{q} \rho_{j}^{q} \tau_{j}^{q}-q \rho_{j} v_{j}\right\} \geq 0, \quad j=1,2, \ldots, n, \\
0 \leq \prod_{j=1}^{n}\left(\sqrt[q]{1+q a_{j} \iota_{j}^{q}+c_{q} \iota_{j}^{q}-q b_{j}}+\sqrt{1+q \rho_{j} \mu_{j} \tau_{j}^{q}+c_{q} \rho_{j}^{q} \tau_{j}^{q}-q \rho_{j} v_{j}}\right)<1
\end{gathered}
$$

Then the problem (SGMQVI) has a solution. Moreover, the solutions set $\Xi$ of (SGMQVI) is a singleton.

Proof. By Theorem 3.1, the problem (SGMQVI) has a solution if and only if (3.1) holds. For the convenience, we define a mapping $F: E \rightarrow E$ by

$$
\begin{gathered}
F(x)=R_{\rho_{1}}^{M_{1}}\left(g_{1}\left(y_{2}(x)\right)-\rho_{1} A_{1}\left(y_{2}(x), x\right)\right), \\
y_{2}(x)=R_{\rho_{2}}^{M_{2}}\left(g_{2}\left(y_{3}(x)\right)-\rho_{2} A_{2}\left(y_{3}(x), y_{2}(x)\right)\right), \\
\vdots \\
y_{n-1}(x)=R_{\rho_{n-1}}^{M_{n-1}}\left(g_{n-1}\left(y_{n}(x)\right)-\rho_{n-1} A_{n-1}\left(y_{n}(x), y_{n-1}(x)\right)\right), \\
y_{n}(x)=R_{\rho_{n}}^{M_{n}}\left(g_{n}(x)-\rho_{n} A_{n}\left(x, y_{n}(x)\right)\right), \quad x \in E .
\end{gathered}
$$

Since $A_{j}: E \times E \rightarrow E$ are $\left(\mu_{j}, v_{j}\right)$-relaxed cocoercive and Lipschitz continuous in the first variable with constant $\tau_{j}$ and $g_{j}: E \rightarrow E$ are $\left(a_{j}, b_{j}\right)$-relaxed cocoercive and Lipschitz continuous with constant $\iota_{j}$ for $j \in\{1,2, \ldots, n\}$, by Theorem 3.2, we know that $F(x)$ and $y_{i}(x)(i=2,3, \ldots, n)$ are continuous on $E$. For any $x, z \in E$,

$$
\begin{aligned}
L_{A_{j}}= & \left\|y_{j+1}(x)-y_{j+1}(z)-\rho_{j}\left[A_{j}\left(y_{j+1}(x), y_{j}(x)\right)-A_{j}\left(y_{j+1}(z), y_{j}(z)\right)\right]\right\|^{q} \\
\leq & -q \rho_{j}\left\langle A_{j}\left(y_{j+1}(x), y_{j}(x)\right)-A_{j}\left(y_{j+1}(z), y_{j}(z)\right), J_{q}\left(y_{j+1}(x)-y_{j+1}(z)\right)\right\rangle \\
& +\left\|y_{j+1}(x)-y_{j+1}(z)\right\|^{q}+c_{q} \rho_{j}^{q}\left\|A_{j}\left(y_{j+1}(x), y_{j}(x)\right)-A_{j}\left(y_{j+1}(z), y_{j}(z)\right)\right\|^{q} \\
\leq & q \rho_{j}\left(\mu_{j}\left\|A_{j}\left(y_{j+1}(x), y_{j}(x)\right)-A_{j}\left(y_{j+1}(z), y_{j}(z)\right)\right\|^{q}-v_{j}\left\|y_{j+1}(x)-y_{j+1}(z)\right\|^{q}\right) \\
& +\left(1+c_{q} \rho_{j}^{q} \tau_{j}^{q}\right)\left\|y_{j+1}(x)-y_{j+1}(z)\right\|^{q}
\end{aligned}
$$




$$
\begin{aligned}
\leq & \left(1+q \rho_{j} \mu_{j} \tau_{j}^{q}+c_{q} \rho_{j}^{q} \tau_{j}^{q}-q \rho_{j} v_{j}\right)\left\|y_{j+1}(x)-y_{j+1}(z)\right\|^{q}, \quad j=2,3, \ldots, n-1, \\
L_{g_{j}}= & \left\|y_{j+1}(x)-y_{j+1}(z)-\left(g_{j}\left(y_{j+1}(x)\right)-g_{j}\left(y_{j+1}(z)\right)\right)\right\|^{q} \\
\leq & \left\|y_{j+1}(x)-y_{j+1}(z)\right\|^{q}-q\left\langle g_{j}\left(y_{j+1}(x)\right)-g_{j}\left(y_{j+1}(z)\right), J_{q}\left(y_{j+1}(x)-y_{j+1}(z)\right)\right\rangle \\
& +c_{q}\left\|g_{j}\left(y_{j+1}(x)\right)-g_{j}\left(y_{j+1}(z)\right)\right\|^{q} \\
\leq & \left(1+q a_{j} \iota_{j}^{q}+c_{q} l_{j}^{q}-q b_{j}\right)\left\|y_{j+1}(x)-y_{j+1}(z)\right\|^{q}, \quad j=2,3, \ldots, n-1, \\
L_{A_{n}}= & \left\|x-z-\rho_{n}\left[A_{n}\left(x, y_{n}(x)\right)-A_{n}\left(z, y_{n}(z)\right)\right]\right\|^{q} \\
\leq & \|x-z\|^{q}-q \rho_{n}\left\langle A_{n}\left(x, y_{n}(x)\right)-A_{n}\left(z, y_{n}(z)\right), J_{q}(x-z)\right\rangle \\
& +c_{q} \rho_{n}^{q}\left\|A_{n}\left(x, y_{n}(x)\right)-A_{n}\left(z, y_{n}(z)\right)\right\|^{q} \\
\leq & \left(1+q \rho_{n} \mu_{n} \tau_{n}^{q}+c_{q} \rho_{n}^{q} \tau_{n}^{q}-q \rho_{n} v_{n}\right)\|x-z\|^{q}, \\
L_{g_{n}}= & \left\|x-z-\left(g_{n}(x)-g_{n}(z)\right)\right\|^{q} \\
\leq & \|x-z\|^{q}-q\left\langle g_{n}(x)-g_{n}(z), J_{q}(x-z)\right\rangle+c_{q}\left\|g_{n}(x)-g_{n}(z)\right\|^{q} \\
\leq & \left(1+q a_{n} \iota_{n}^{q}+c_{q} \iota_{n}^{q}-q b_{n}\right)\|x-z\|^{q}, \\
L_{A_{1}}= & \left\|y_{2}(x)-y_{2}(z)-\rho_{1}\left[A_{1}\left(y_{2}(x), x\right)-A_{1}\left(y_{2}(z), z\right)\right]\right\|^{q} \\
\leq & \left(1+q \rho_{1} \mu_{1} \tau_{1}^{q}+c_{q} \rho_{1}^{q} \tau_{1}^{q}-q \rho_{1} v_{1}\right)\left\|y_{2}(x)-y_{2}(z)\right\|^{q}, \\
L_{g_{1}}= & \left\|y_{2}(x)-y_{2}(z)-\left(g_{1}\left(y_{2}(x)\right)-g_{1}\left(y_{2}(z)\right)\right)\right\|^{q} \\
\leq & \left(1+q a_{1} \iota_{1}^{q}+c_{q} \iota_{1}^{q}-q b_{1}\right)\left\|y_{2}(x)-y_{2}(z)\right\|^{q} .
\end{aligned}
$$

From Lemma 2.10, it yields that

$$
\begin{aligned}
&\|F(x)-F(z)\| \\
&=\left\|R_{\rho_{1}}^{M_{1}}\left(g_{1}\left(y_{2}(x)\right)-\rho_{1} A_{1}\left(y_{2}(x), x\right)\right)-R_{\rho_{1}}^{M_{1}}\left(g_{1}\left(y_{2}(z)\right)-\rho_{1} A_{1}\left(y_{2}(z), z\right)\right)\right\| \\
& \leq\left\|\left(g_{1}\left(y_{2}(x)\right)-\rho_{1} A_{1}\left(y_{2}(x), x\right)\right)-\left(g_{1}\left(y_{2}(z)\right)-\rho_{1} A_{1}\left(y_{2}(z), z\right)\right)\right\| \\
&=\left\|\left(g_{1}\left(y_{2}(x)\right)-g_{1}\left(y_{2}(z)\right)\right)-\rho_{1}\left(A_{1}\left(y_{2}(x), x\right)-A_{1}\left(y_{2}(z), z\right)\right)\right\| \\
& \leq\left\|\left(y_{2}(x)-y_{2}(z)\right)-\rho_{1}\left(A_{1}\left(y_{2}(x), x\right)-A_{1}\left(y_{2}(z), z\right)\right)\right\| \\
&+\left\|\left(y_{2}(x)-y_{2}(z)\right)-\left(g_{1}\left(y_{2}(x)\right)-g_{1}\left(y_{2}(z)\right)\right)\right\| \\
& \leq\left(\sqrt[q]{1+q a_{1} l_{1}^{q}+c_{q} l_{1}^{q}-q b_{1}}+\sqrt[q]{1+q \rho_{1} \mu_{1} \tau_{1}^{q}+c_{q} \rho_{1}^{q} \tau_{1}^{q}-q \rho_{1} v_{1}}\right)\left\|y_{2}(x)-y_{2}(z)\right\| .
\end{aligned}
$$


Note that, for each $j \in\{2,3, \ldots, n-1\}$,

$$
\begin{aligned}
&\left\|y_{j}(x)-y_{j}(z)\right\| \\
&=\left\|R_{\rho_{j}}^{M_{j}}\left(g_{j}\left(y_{j+1}(x)\right)-\rho_{j} A_{j}\left(y_{j+1}(x), y_{j}(x)\right)\right)-R_{\rho_{j}}^{M_{j}}\left(g_{j}\left(y_{j+1}(z)\right)-\rho_{j} A_{j}\left(y_{j+1}(z), y_{j}(z)\right)\right)\right\| \\
& \leq\left\|\left(g_{j}\left(y_{j+1}(x)\right)-\rho_{j} A_{j}\left(y_{j+1}(x), y_{j}(x)\right)\right)-\left(g_{j}\left(y_{j+1}(z)\right)-\rho_{j} A_{j}\left(y_{j+1}(z), y_{j}(z)\right)\right)\right\| \\
&=\left\|\left(g_{j}\left(y_{j+1}(x)\right)-g_{j}\left(y_{j+1}(z)\right)\right)-\rho_{j}\left(A_{j}\left(y_{j+1}(x), y_{j}(x)\right)-A_{j}\left(y_{j+1}(z), y_{j}(z)\right)\right)\right\| \\
& \leq\left\|\left(y_{j+1}(x)-y_{j+1}(z)\right)-\rho_{j}\left(A_{j}\left(y_{j+1}(x), y_{j}(x)\right)-A_{j}\left(y_{j+1}(z), y_{j}(z)\right)\right)\right\| \\
&+\left\|\left(y_{j+1}(x)-y_{j+1}(z)\right)-\left(g_{j}\left(y_{j+1}(x)\right)-g_{j}\left(y_{j+1}(z)\right)\right)\right\| \\
& \leq\left(\sqrt[q]{1+q \rho_{j} \mu_{j} \tau_{j}^{q}+c_{q} \rho_{j}^{q} \tau_{j}^{q}-q \rho_{j} v_{j}}+\sqrt[q]{1+q a_{j} l_{j}^{q}+c_{q} l_{j}^{q}-q b_{j}}\right)\left\|y_{j+1}(x)-y_{j+1}(z)\right\|, \\
&\left\|y_{n}(x)-y_{n}(z)\right\| \\
&=\left\|R_{\rho_{n}}^{M_{n}}\left(g_{n}(x)-\rho_{n} A_{n}\left(x, y_{n}(x)\right)\right)-R_{\rho_{n}}^{M_{n}}\left(g_{n}(z)-\rho_{n} A_{n}\left(z, y_{n}(z)\right)\right)\right\| \\
& \leq\left\|\left(g_{n}(x)-g_{n}(z)\right)-\rho_{n}\left(A_{n}\left(x, y_{n}(x)\right)-A_{n}\left(z, y_{n}(z)\right)\right)\right\| \\
& \leq\left\|x-z-\rho_{n}\left(A_{n}\left(x, y_{n}(x)\right)-A_{n}\left(z, y_{n}(z)\right)\right)\right\|+\left\|x-z-\left(g_{n}(x)-g_{n}(z)\right)\right\| \\
& \leq\left(\sqrt[q]{1+q \rho_{n} \mu_{n} \tau_{n}^{q}+c_{q} \rho_{n}^{q} \tau_{n}^{q}-q \rho_{n} v_{n}}+\sqrt[q]{1+q a_{n} l_{n}^{q}+c_{q} l_{n}^{q}-q b_{n}}\right)\|x-z\| .
\end{aligned}
$$

Therefore, we obtain

$$
\|F(x)-F(z)\| \leq \prod_{j=1}^{n}\left(\sqrt[q]{1+q \rho_{j} \mu_{j} \tau_{j}^{q}+c_{q} \rho_{j}^{q} \tau_{j}^{q}-q \rho_{j} v_{j}}+\sqrt[q]{1+q a_{j} l_{j}^{q}+c_{q} \iota_{j}^{q}-q b_{j}}\right)\|x-z\| .
$$

It follows from (3.12) that the mapping $F$ is contractive. By banach's contraction principle, there exists a unique $x_{1}^{*} \in E$ such that $F\left(x_{1}^{*}\right)=x_{1}^{*}$. Therefore, by Theorem 3.2, there exists an unique $\left(x_{1}^{*}, x_{2}^{*}, \ldots, x_{n}^{*}\right) \in E^{n}$ such that $\left(x_{1}^{*}, x_{2}^{*}, \ldots, x_{n}^{*}\right)$ is a solution of the problem (SGMQVI), where $x_{i}^{*}=y_{i}\left(x_{1}^{*}\right)$ for $i=2,3, \ldots, n$, that is, $\Xi=\left\{\left(x_{1}^{*}, x_{2}^{*}, \ldots, x_{n}^{*}\right)\right\}$. This completes the proof.

\section{Convergence Analysis}

In this section, we introduce several implicit algorithms with errors and explicit algorithms without errors for solving the system of generalized mixed quasivariational inclusions problem (SGMQVI) and then explore the convergence analysis of the iterative sequences generated by the corresponding algorithms.

From Section 3, we know that the system of generalized mixed quasivariational inclusions problem (SGMQVI) is equivalent to the fixed point problem (3.1). This equivalent formulation is crucial from the numerical analysis point of view. As we know, this fixed 
point formulation has been used to suggest and analyze some iterative methods for solving variational inequalities and related optimization problems. By using the relations between the problem (SGMQVI) and the fixed point problem (3.1), we construct the following iterative algorithms for solving the system of generalized mixed quasivariational inclusions problem (2.3).

Algorithm 4.1. Let $\rho_{j}$ be positive constants for all $j=1,2, \ldots, n$. For any given points $x_{1,0} \in E$, define sequences $\left\{x_{j, k}\right\} j=1,2, \ldots, n$ in $E$ by the following implicit algorithm:

$$
\begin{gathered}
x_{n, k}=R_{\rho_{n}}^{M_{n}}\left(g_{n}\left(x_{1, k}\right)-\rho_{n} A_{n}\left(x_{1, k}, x_{n, k}\right)\right), \\
x_{n-1, k}=R_{\rho_{n-1}}^{M_{n-1}}\left(g_{n-1}\left(x_{n, k}\right)-\rho_{n-1} A_{n-1}\left(x_{n, k}, x_{n-1, k}\right)\right), \\
\vdots \\
x_{2, k}=R_{\rho_{2}}^{M_{2}}\left(g_{2}\left(x_{3, k}\right)-\rho_{2} A_{2}\left(x_{3, k}, x_{2, k}\right)\right), \\
x_{1, k+1}=\left(1-\alpha_{k}\right) x_{1, k}+\alpha_{k} R_{\rho_{1}}^{M_{1}}\left(g_{1}\left(x_{2, k}\right)-\rho_{1} A_{1}\left(x_{2, k}, x_{1, k}\right)\right)+e_{k}, \quad k=0,1,2, \ldots,
\end{gathered}
$$

where $\left\{e_{k}\right\} \subset E$ and $\left\{\alpha_{k}\right\}$ is a real sequence in $[0,1]$.

If $e_{k} \equiv 0$ for all $k \geq 0$, then Algorithm 4.1 is reduced to the following result.

Algorithm 4.2. Let $\rho_{j}$ be positive constants for all $j=1,2, \ldots, n$. For any given points $x_{1,0} \in E$, define sequences $\left\{x_{j, k}\right\} j=1,2, \ldots, n$ in $E$ by the following implicit algorithm

$$
\begin{gathered}
x_{n, k}=R_{\rho_{n}}^{M_{n}}\left(g_{n}\left(x_{1, k}\right)-\rho_{n} A_{n}\left(x_{1, k}, x_{n, k}\right)\right), \\
x_{n-1, k}=R_{\rho_{n-1}}^{M_{n-1}}\left(g_{n-1}\left(x_{n, k}\right)-\rho_{n-1} A_{n-1}\left(x_{n, k}, x_{n-1, k}\right)\right), \\
\vdots \\
x_{2, k}=R_{\rho_{2}}^{M_{2}}\left(g_{2}\left(x_{3, k}\right)-\rho_{2} A_{2}\left(x_{3, k}, x_{2, k}\right)\right), \\
x_{1, k+1}=\left(1-\alpha_{k}\right) x_{1, k}+\alpha_{k} R_{\rho_{1}}^{M_{1}}\left(g_{1}\left(x_{2, k}\right)-\rho_{1} A_{1}\left(x_{2, k}, x_{1, k}\right)\right), \quad k=0,1,2, \ldots,
\end{gathered}
$$

where $\left\{\alpha_{k}\right\}$ is a real sequence in $[0,1]$.

Now we construct an explicit algorithms for solving the system of generalized mixed quasivariational inclusions problem (SGMQVI). 
Algorithm 4.3. Let $\rho_{j}$ be positive constants for all $j=1,2, \ldots, n$. For any given points $\left(x_{1,0}, x_{2,0}\right.$, $\left.\ldots, x_{n-1,0}, x_{n, 0}\right) \in E^{n}$, define sequences $\left\{x_{j, k}\right\}(j=1,2, \ldots, n)$ in $E$ by the following explicit algorithm

$$
\begin{gathered}
x_{n, k+1}=R_{\rho_{n}}^{M_{n}}\left(g_{n}\left(x_{1, k}\right)-\rho_{n} A_{n}\left(x_{1, k}, x_{n, k}\right)\right), \\
x_{n-1, k+1}=R_{\rho_{n-1}}^{M_{n-1}}\left(g_{n-1}\left(x_{n, k+1}\right)-\rho_{n-1} A_{n-1}\left(x_{n, k+1}, x_{n-1, k}\right)\right), \\
\vdots \\
x_{2, k+1}=R_{\rho_{2}}^{M_{2}}\left(g_{2}\left(x_{3, k+1}\right)-\rho_{2} A_{2}\left(x_{3, k+1}, x_{2, k}\right)\right), \\
x_{1, k+1}=\left(1-\alpha_{k}\right) x_{1, k}+\alpha_{k} R_{\rho_{1}}^{M_{1}}\left(g_{1}\left(x_{2, k+1}\right)-\rho_{1} A_{1}\left(x_{2, k+1}, x_{1, k}\right)\right), \quad k=0,1,2, \ldots,
\end{gathered}
$$

where $\left\{\alpha_{k}\right\}$ is a real sequence in $[0,1]$.

Remark 4.4. If $n=2, E=H$ is a Hilbert space, and $K$ is a closed convex subset of $E, \phi(x)=$ $\delta_{K}(x)$ for all $x \in K$, and $M_{1}(x)=M_{2}(x)=\partial \phi(x)$ for all $x \in E$, where $\delta_{K}$ is the indicator function of $K$, and $\partial \phi$ denotes the subdifferential operator of $\phi$, then, from Remark 2.9, Algorithms 4.1 and 4.3 are reduced to the Algorithms 4.5 and 4.6 for solving the system of general variational inequalities problem (2.7).

Algorithm 4.5. Let $\rho_{j}$ be positive constants for all $j=1$, 2 . For any given points $x_{1,0} \in E$, define sequences $\left\{x_{j, k}\right\} j=1,2$ in $E$ by the following implicit algorithm:

$$
\begin{gathered}
x_{2, k}=P_{K}\left(g_{2}\left(x_{1, k}\right)-\rho_{2} A_{2}\left(x_{1, k}, x_{2, k}\right)\right), \\
x_{1, k+1}=\left(1-\alpha_{k}\right) x_{1, k}+\alpha_{k} P_{K}\left(g_{1}\left(x_{2, k}\right)-\rho_{1} A_{1}\left(x_{2, k}, x_{1, k}\right)\right)+e_{k}, \quad k=0,1,2, \ldots,
\end{gathered}
$$

where $\left\{e_{k}\right\} \subset E$ and $\left\{\alpha_{k}\right\}$ is a real sequence in $[0,1]$.

Algorithm 4.6. Let $\rho_{j}$ be positive constants for all $j=1$, 2 . For any given points $\left(x_{1,0}, x_{2,0}\right) \in E^{2}$, define sequences $\left\{x_{j, k}\right\}(j=1,2)$ in $E$ by the following explicit algorithm:

$$
\begin{gathered}
x_{2, k+1}=P_{K}\left(g_{2}\left(x_{1, k}\right)-\rho_{2} A_{2}\left(x_{1, k}, x_{2, k}\right)\right), \\
x_{1, k+1}=\left(1-\alpha_{k}\right) x_{1, k}+\alpha_{k} P_{K}\left(g_{1}\left(x_{2, k+1}\right)-\rho_{1} A_{1}\left(x_{2, k+1}, x_{1, k}\right)\right), \quad k=0,1,2, \ldots,
\end{gathered}
$$

where $\left\{\alpha_{k}\right\}$ is a real sequence in $[0,1]$.

Theorem 4.7. Let $E$ be a real q-uniformly smooth Banach space, and let $M_{j}, A_{j}$, and $g_{j}(j=$ $1,2, \ldots, n)$ be the same as in Theorem 3.4. Assume that $\left\{\alpha_{n}\right\}$ is a real sequence in $(0,1]$ and satisfy the following conditions:

(i) $\sum_{k=0}^{\infty} \alpha_{k}=\infty$;

(ii) $\sum_{k=0}^{\infty}\left\|e_{k}\right\|<+\infty$; 
(iii) $\min \left\{1+q a_{j} \iota_{j}^{q}+c_{q} \iota_{j}^{q}-q b_{j}, 1+q \rho_{j} \mu_{j} \tau_{j}^{q}+c_{q} \rho_{j}^{q} \tau_{j}^{q}-q \rho_{j} v_{j}\right\} \geq 0, j=1,2, \ldots, n$;

(iv) $0<\prod_{j=1}^{n}\left(\sqrt[q]{1+q a_{j} q_{j}^{q}+c_{q} l_{j}^{q}-q b_{j}}+\sqrt[q]{1+q \rho_{j} \mu_{j} \tau_{j}^{q}+c_{q} \rho_{j}^{q} \tau_{j}^{q}-q \rho_{j} v_{j}}\right)<1$.

Then the sequences $\left\{x_{j, k}\right\}(j=1,2, \ldots, n)$ generated by Algorithm 4.1 converge strongly to $x_{j}^{*}(j=$ $1,2, \ldots, n)$, respectively, such that $\left(x_{1}^{*}, x_{2}^{*}, \ldots, x_{n}^{*}\right) \in \Xi$.

Proof. By Theorem 3.4, we know that there exist an unique point $\left(x_{1}^{*}, x_{2}^{*}, \ldots, x_{n}^{*}\right) \in E^{n}$ such that $\left(x_{1}^{*}, x_{2}^{*}, \ldots, x_{n}^{*}\right) \in \Xi$. Then, from Theorem 3.1, one has

$$
\begin{gathered}
x_{1}^{*}=R_{\rho_{1}}^{M_{1}}\left(g_{1}\left(x_{2}^{*}\right)-\rho_{1} A_{1}\left(x_{2}^{*}, x_{1}^{*}\right)\right), \\
x_{2}^{*}=R_{\rho_{2}}^{M_{2}}\left(g_{2}\left(x_{3}^{*}\right)-\rho_{2} A_{2}\left(x_{3}^{*}, x_{2}^{*}\right)\right), \\
\vdots \\
x_{n-1}^{*}=R_{\rho_{n-1}}^{M_{n-1}}\left(g_{n-1}\left(x_{n}^{*}\right)-\rho_{n-1} A_{n-1}\left(x_{n}^{*}, x_{n-1}^{*}\right)\right), \\
x_{n}^{*}=R_{\rho_{n}}^{M_{n}}\left(g_{n}\left(x_{1}^{*}\right)-\rho_{n} A_{n}\left(x_{1}^{*}, x_{n}^{*}\right)\right) .
\end{gathered}
$$

Therefore, from both (4.1) and (4.6), we have

$$
\begin{aligned}
\left\|x_{1, k+1}-x_{1}^{*}\right\|= & \left\|\left(1-\alpha_{k}\right) x_{1, k}+\alpha_{k} R_{\rho_{1}}^{M_{1}}\left(g_{1}\left(x_{2, k}\right)-\rho_{1} A_{1}\left(x_{2, k}, x_{1, k}\right)\right)+e_{k}-x_{1}^{*}\right\| \\
= & \left\|\left(1-\alpha_{k}\right)\left(x_{1, k}-x_{1}^{*}\right)+\alpha_{k}\left(R_{\rho_{1}}^{M_{1}}\left(g_{1}\left(x_{2, k}\right)-\rho_{1} A_{1}\left(x_{2, k}, x_{1, k}\right)\right)-x_{1}^{*}\right)+e_{k}\right\| \\
= & \| \alpha_{k}\left(R_{\rho_{1}}^{M_{1}}\left(g_{1}\left(x_{2, k}\right)-\rho_{1} A_{1}\left(x_{2, k}, x_{1, k}\right)\right)-R_{\rho_{1}}^{M_{1}}\left(g_{1}\left(x_{2}^{*}\right)-\rho_{1} A_{1}\left(x_{2}^{*}, x_{1}^{*}\right)\right)\right) \\
& +\left(1-\alpha_{k}\right)\left(x_{1, k}-x_{1}^{*}\right)+e_{k} \| \\
\leq & \alpha_{k}\left\|R_{\rho_{1}}^{M_{1}}\left(g_{1}\left(x_{2, k}\right)-\rho_{1} A_{1}\left(x_{2, k}, x_{1, k}\right)\right)-R_{\rho_{1}}^{M_{1}}\left(g_{1}\left(x_{2}^{*}\right)-\rho_{1} A_{1}\left(x_{2}^{*}, x_{1}^{*}\right)\right)\right\| \\
& +\left(1-\alpha_{k}\right)\left\|x_{1, k}-x_{1}^{*}\right\|+\left\|e_{k}\right\| \\
\leq & \alpha_{k}\left\|\left(g_{1}\left(x_{2, k}\right)-\rho_{1} A_{1}\left(x_{2, k}, x_{1, k}\right)\right)-\left(g_{1}\left(x_{2}^{*}\right)-\rho_{1} A_{1}\left(x_{2}^{*}, x_{1}^{*}\right)\right)\right\| \\
& +\left(1-\alpha_{k}\right)\left\|x_{1, k}-x_{1}^{*}\right\|+\left\|e_{k}\right\| \\
= & \alpha_{k}\left\|\left(g_{1}\left(x_{2, k}\right)-g_{1}\left(x_{2}^{*}\right)\right)-\left(\rho_{1} A_{1}\left(x_{2, k}, x_{1, k}\right)-\rho_{1} A_{1}\left(x_{2}^{*}, x_{1}^{*}\right)\right)\right\| \\
& +\left(1-\alpha_{k}\right)\left\|x_{1, k}-x_{1}^{*}\right\|+\left\|e_{k}\right\| \\
\leq & \left(1-\alpha_{k}\right)\left\|x_{1, k}-x_{1}^{*}\right\|+\left\|e_{k}\right\|+\alpha_{k}\left\|\left(x_{2, k}-x_{2}^{*}\right)-\left(g_{1}\left(x_{2, k}\right)-g_{1}\left(x_{2}^{*}\right)\right)\right\| \\
& +\alpha_{k}\left\|\left(x_{2, k}-x_{2}^{*}\right)-\rho_{1}\left(A_{1}\left(x_{2, k}, x_{1, k}\right)-A_{1}\left(x_{2}^{*}, x_{1}^{*}\right)\right)\right\| .
\end{aligned}
$$


Since $A_{j}: E \times E \rightarrow E$ are $\left(\mu_{j}, v_{j}\right)$-relaxed cocoercive and Lipschitz continuous in the first variable with constant $\tau_{j}$ and $g_{j}: E \rightarrow E$ are $\left(a_{j}, b_{j}\right)$-relaxed cocoercive and Lipschitz continuous with constant $\iota_{j}$ for $j \in\{1,2, \ldots, n\}$, we can conclude

$$
\begin{aligned}
& L_{A_{i}}^{*}=\left\|x_{i+1, k}-x_{i+1}^{*}-\rho_{\mathrm{i}}\left[A_{i}\left(x_{i+1, k}, x_{i, k}\right)-A_{i}\left(x_{i+1}^{*}, x_{i}^{*}\right)\right]\right\|^{q} \\
& \leq-q \rho_{i}\left\langle A_{i}\left(x_{i+1, k}, x_{i, k}\right)-A_{i}\left(x_{i+1}^{*}, x_{i}^{*}\right), J_{q}\left(x_{i+1, k}-x_{i+1}^{*}\right)\right\rangle \\
& +\left\|x_{i+1, k}-x_{i+1}^{*}\right\|^{q}+c_{q} \rho_{i}^{q}\left\|A_{i}\left(x_{i+1, k}, x_{i, k}\right)-A_{i}\left(x_{i+1}^{*}, x_{i}^{*}\right)\right\|^{q} \\
& \leq q \rho_{i}\left(\mu_{i}\left\|A_{i}\left(x_{i+1, k}, x_{i, k}\right)-A_{i}\left(x_{i+1}^{*}, x_{i}^{*}\right)\right\|^{q}-v_{i}\left\|x_{i+1, k}-x_{i+1}^{*}\right\|^{q}\right) \\
& +\left(1+c_{q} \rho_{i}^{q} \tau_{i}^{q}\right)\left\|x_{i+1, k}-x_{i+1}^{*}\right\|^{q} \\
& \leq\left(1+q \rho_{i} \mu_{i} \tau_{i}^{q}+c_{q} \rho_{i}^{q} \tau_{i}^{q}-q \rho_{i} v_{i}\right)\left\|x_{i+1, k}-x_{i+1}^{*}\right\|^{q}, \quad i=1,2, \ldots, n-1, \\
& L_{g_{i}}^{*}=\left\|x_{i+1, k}-x_{i+1}^{*}-\left(g_{i}\left(x_{i+1, k}\right)-g_{i}\left(x_{i+1}^{*}\right)\right)\right\|^{q} \\
& \leq-q\left\langle g_{i}\left(x_{i+1, k}\right)-g_{i}\left(x_{i+1}^{*}\right), J_{q}\left(x_{i+1, k}-x_{i+1}^{*}\right)\right\rangle \\
& +\left\|x_{i+1, k}-x_{i+1}^{*}\right\|^{q}+c_{q}\left\|g_{i}\left(x_{i+1, k}\right)-g_{i}\left(x_{i+1}^{*}\right)\right\|^{q} \\
& \leq\left(1+q a_{i} \iota_{i}^{q}+c_{q} l_{i}^{q}-q b_{i}\right)\left\|x_{i+1, k}-x_{i+1}^{*}\right\|^{q}, \quad i=1,2, \ldots, n-1, \\
& L_{A_{n}}^{*}=\left\|x_{1, k}-x_{1}^{*}-\rho_{n}\left[A_{n}\left(x_{1, k}, x_{n, k}\right)-A_{n}\left(x_{1}^{*}, x_{n}^{*}\right)\right]\right\|^{q} \\
& \leq\left\|x_{1, k}-x_{1}^{*}\right\|^{q}-q \rho_{n}\left\langle A_{n}\left(x_{1, k}, x_{n, k}\right)-A_{n}\left(x_{1}^{*}, x_{n}^{*}\right), J_{q}\left(x_{1, k}-x_{1}^{*}\right)\right\rangle \\
& +c_{q} \rho_{n}^{q}\left\|A_{n}\left(x_{1, k}, x_{n, k}\right)-A_{n}\left(x_{1}^{*}, x_{n}^{*}\right)\right\|^{q} \\
& \leq\left(1+q \rho_{n} \mu_{n} \tau_{n}^{q}+c_{q} \rho_{n}^{q} \tau_{n}^{q}-q \rho_{n} v_{n}\right)\left\|x_{1, k}-x_{1}^{*}\right\|^{q} ， \\
& L_{g_{n}}^{*}=\left\|x_{1, k}-x_{1}^{*}-\left(g_{n}\left(x_{1, k}\right)-g_{n}\left(x_{1}^{*}\right)\right)\right\|^{q} \\
& \leq\left\|x_{1, k}-x_{1}^{*}\right\|^{q}-q\left\langle g_{n}\left(x_{1, k}\right)-g_{n}\left(x_{1}^{*}\right), J_{q}\left(x_{1, k}-x_{1}^{*}\right)\right\rangle+c_{q}\left\|g_{n}\left(x_{1, k}\right)-g_{n}\left(x_{1}^{*}\right)\right\|^{q} \\
& \leq\left(1+q a_{n} \iota_{n}^{q}+c_{q} \iota_{n}^{q}-q b_{n}\right)\left\|x_{1, k}-x_{1}^{*}\right\|^{q} \text {. }
\end{aligned}
$$

Noticing that, for each $i \in\{1,2, \ldots, n-2\}$,

$$
\begin{aligned}
\left\|x_{i+1, k}-x_{i+1}^{*}\right\|= & \| R_{\rho_{i+1}}^{M_{i+1}}\left(g_{i+1}\left(x_{i+2, k}\right)-\rho_{i+1} A_{i+1}\left(\mathrm{x}_{i+2, k}, x_{i+1, k}\right)\right) \\
& -R_{\rho_{i+1}}^{M_{i+1}}\left(g_{i+1}\left(x_{i+2}^{*}\right)-\rho_{i+1} A_{i+1}\left(x_{i+2}^{*}, x_{i+1}^{*}\right)\right) \| \\
\leq & \|\left(g_{i+1}\left(x_{i+2, k}\right)-\rho_{i+1} A_{i+1}\left(x_{i+2, k}, x_{i+1, k}\right)\right) \\
& -\left(g_{i+1}\left(x_{i+2}^{*}\right)-\rho_{i+1} A_{i+1}\left(x_{i+2}^{*}, x_{i+1}^{*}\right)\right) \|
\end{aligned}
$$




$$
\begin{aligned}
= & \left\|\left(g_{i+1}\left(x_{i+2, k}\right)-g_{i+1}\left(x_{i+2}^{*}\right)\right)-\rho_{i+1}\left(A_{i+1}\left(x_{i+2, k}, x_{i+1, k}\right)-A_{i+1}\left(x_{i+2}^{*}, x_{i+1}^{*}\right)\right)\right\| \\
\leq & \left\|\left(x_{i+2, k}-x_{i+2}^{*}\right)-\rho_{i+1}\left(A_{i+1}\left(x_{i+2, k}, x_{i+1, k}\right)-A_{i+1}\left(x_{i+2}^{*}, x_{i+1}^{*}\right)\right)\right\| \\
& +\left\|\left(x_{i+2, k}-x_{i+2}^{*}\right)-\left(g_{i+1}\left(x_{i+2, k}\right)-g_{i+1}\left(x_{i+2}^{*}\right)\right)\right\| \\
\leq & \left(\sqrt[q]{1+q \rho_{i+1} \mu_{i+1} \tau_{i+1}^{q}+c_{q} \rho_{i+1}^{q} \tau_{i+1}^{q}-q \rho_{i+1} v_{i+1}}\right. \\
& \left.+\sqrt[9]{1+q a_{i+1} t_{i+1}^{q}+c_{q} l_{i+1}^{q}-q b_{i+1}}\right)\left\|x_{i+2, k}-x_{i+2}^{*}\right\|, \\
\left\|x_{n, k}-x_{n}^{*}\right\|= & \left\|R_{\rho_{n}}^{M_{n}}\left(g_{n}\left(x_{1, k}\right)-\rho_{n} A_{n}\left(x_{1, k}, x_{n, k}\right)\right)-R_{\rho_{n}}^{M_{n}}\left(g_{n}\left(x_{1}^{*}\right)-\rho_{n} A_{n}\left(x_{1}^{*}, x_{n}^{*}\right)\right)\right\| \\
\leq & \left\|\left(g_{n}\left(x_{1, k}\right)-\rho_{n} A_{n}\left(x_{1, k}, x_{n, k}\right)\right)-\left(g_{n}\left(x_{1}^{*}\right)-\rho_{n} A_{n}\left(x_{1}^{*}, x_{n}^{*}\right)\right)\right\| \\
= & \left\|\left(g_{n}\left(x_{1, k}\right)-g_{n}\left(x_{1}^{*}\right)\right)-\rho_{n}\left(A_{n}\left(x_{1, k}, x_{n, k}\right)-A_{n}\left(x_{1}^{*}, x_{n}^{*}\right)\right)\right\| \\
\leq & \left\|\left(x_{1, k}-x_{1}^{*}\right)-\left(\rho_{n} A_{n}\left(x_{1, k}, x_{n, k}\right)-\rho_{n} A_{n}\left(x_{1}^{*}, x_{n}^{*}\right)\right)\right\| \\
& +\left\|\left(x_{1, k}-x_{1}^{*}\right)-\left(g_{n}\left(x_{1, k}\right)-g_{n}\left(x_{1}^{*}\right)\right)\right\| \\
\leq & \left(\sqrt[q]{1+q \rho_{n} \mu_{n} \tau_{n}^{q}+c_{q} \rho_{n}^{q} \tau_{n}^{q}-q \rho_{n} v_{n}}+\sqrt[q^{q}]{1+q a_{n} l_{n}^{q}+c_{q} l_{n}^{q}-q b_{n}}\right) \\
& \times\left\|x_{1, k}-x_{1}^{*}\right\| .
\end{aligned}
$$

As a consequence, we have

$$
\begin{aligned}
\left\|x_{1, k+1}-x_{1}^{*}\right\| \leq & \alpha_{k} \prod_{j=1}^{n}\left(\sqrt[9]{1+q \rho_{j} \mu_{j} \tau_{j}^{q}+c_{q} \rho_{j}^{q} \tau_{j}^{q}-q \rho_{j} v_{j}}+\sqrt[9]{1+q a_{j} l_{j}^{q}+c_{q} l_{j}^{q}-q b_{j}}\right)\left\|x_{1, k}-x_{1}^{*}\right\| \\
& +\left(1-\alpha_{k}\right)\left\|x_{1, k}-x_{1}^{*}\right\|+\left\|e_{k}\right\| \\
= & {\left[1-\alpha_{k}\left(1-\prod_{j=1}^{n}\left(\sqrt[q]{1+q \rho_{j} \mu_{j} \tau_{j}^{q}+c_{q} \rho_{j}^{q} \tau_{j}^{q}-q \rho_{j} v_{j}}+\sqrt[q]{1+q a_{j} \iota_{j}^{q}+c_{q} l_{j}^{q}-q b_{j}}\right)\right)\right] } \\
& \times\left\|x_{1, k}-x_{1}^{*}\right\|+\left\|e_{k}\right\| .
\end{aligned}
$$

Putting $\lambda_{k}=\alpha_{k}\left(1-\prod_{j=1}^{n}\left(\sqrt[q]{1+q \rho_{j} \mu_{j} \tau_{j}^{q}+c_{q} \rho_{j}^{q} \tau_{j}^{q}-q \rho_{j} v_{j}}+\sqrt[q]{1+q a_{j} l_{j}^{q}+c_{q} l_{j}^{q}-q b_{j}}\right)\right), C_{k}=$ $0, \quad B_{k}=\left\|x_{1, k}-x_{1}^{*}\right\|$, and $D_{k}=\left\|e_{k}\right\|$. Then $B_{k+1} \leq\left(1-\lambda_{k}\right) B_{k}+C_{k}+D_{k}$. From the conditions (i)(iv), it follows that

$$
\sum_{k=0}^{\infty} \lambda_{k}=\infty, \quad C_{k}=0\left(\lambda_{k}\right), \quad \sum_{k=0}^{\infty} D_{k}<\infty, \quad 0<\lambda_{k}<1, \forall k \in N
$$


Journal of Applied Mathematics

Therefore, by Lemma 2.11 and (4.11), one has

$$
\lim _{k \rightarrow \infty} B_{k}=0
$$

that is, $\lim _{k \rightarrow \infty} x_{1, k}=x_{1}^{*}$. Again from (iii), this shows that

$$
\sqrt[q]{1+q \rho_{j} \mu_{j} \tau_{j}^{q}+c_{q} \rho_{j}^{q} \tau_{j}^{q}-q \rho_{j} \nu_{j}}+\sqrt[q]{1+q a_{j} \iota_{j}^{q}+c_{q} \iota_{j}^{q}-q b_{j}} \geq 0, \quad j=2,3, \ldots, n,
$$

and so,

$$
\lim _{k \rightarrow \infty}\left\|x_{j, k}-x_{j}^{*}\right\|=0
$$

That is, $x_{j, k} \rightarrow x_{j}^{*}$ as $k \rightarrow \infty$ for $j=2,3, \ldots, n$. Thus, $\left(x_{1, k}, x_{2, k}, \ldots, x_{n, k}\right)$ converges strongly to $\left(x_{1}^{*}, x_{2}^{*}, \ldots, x_{n}^{*}\right)$. This completes the proof.

Theorem 4.8. Let E be a real q-uniformly smooth Banach space, and let $M_{j}, A_{j}$ and $g_{j}(j=1,2, \ldots$, $n)$ be the same as in Theorem 3.4. Assume that $\left\{\alpha_{n}\right\}$ is a real sequence in $(0,1]$ and satisfies the following conditions:

(i) $\sum_{k=0}^{\infty} \alpha_{k}=\infty$;

(ii) $\min \left\{1+q a_{j} l_{j}^{q}+c_{q} \iota_{j}^{q}-q b_{j}, 1+q \rho_{j} \mu_{j} \tau_{j}^{q}+c_{q} \rho_{j}^{q} \tau_{j}^{q}-q \rho_{j} v_{j}\right\} \geq 0, j=1,2, \ldots, n$;

(iii) $0<\prod_{j=1}^{n}\left(\sqrt[q]{1+q a_{j} l_{j}^{q}+c_{q} l_{j}^{q}-q b_{j}}+\sqrt[q]{1+q \rho_{j} \mu_{j} \tau_{j}^{q}+c_{q} \rho_{j}^{q} \tau_{j}^{q}-q \rho_{j} v_{j}}\right)<1$.

Then the sequences $\left\{x_{j, k}\right\}(j=1,2, \ldots, n)$ generated by Algorithm 4.2 converge strongly to $x_{j}^{*}(j=$ $1,2, \ldots, n)$, respectively, such that $\left(x_{1}^{*}, x_{2}^{*}, \ldots, x_{n}^{*}\right) \in \Xi$.

Proof. It directly follows from Theorem 4.7, and so the proof is omitted. This completes the proof.

Theorem 4.9. Let $E$ be a real q-uniformly smooth Banach space, and let $M_{j}, A_{j}$ and $g_{j}(j=1,2, \ldots$, $n)$ be the same as in Theorem 3.4. Assume that $\left\{\alpha_{n}\right\}$ is a real sequence in $(0,1]$ and satisfy the following conditions:

(i) $\sum_{k=0}^{\infty} \alpha_{k}=\infty$;

(ii) $\min \left\{1+q a_{j} \iota_{j}^{q}+c_{q} \iota_{j}^{q}-q b_{j}, 1+q \rho_{j} \mu_{j} \tau_{j}^{q}+c_{q} \rho_{j}^{q} \tau_{j}^{q}-q \rho_{j} v_{j}\right\} \geq 0, j=1,2, \ldots, n$;

(iii) $0<\prod_{j=1}^{n}\left(\sqrt[q]{1+q a_{j} \iota_{j}^{q}+c_{q} l_{j}^{q}-q b_{j}}+\sqrt[q]{1+q \rho_{j} \mu_{j} \tau_{j}^{q}+c_{q} \rho_{j}^{q} \tau_{j}^{q}-q \rho_{j} v_{j}}\right)<1$. 
Then the sequences $\left\{x_{j, k}\right\}(j=1,2, \ldots, n)$ generated by Algorithm 4.3 converge strongly to $x_{j}^{*}(j=$ $1,2, \ldots, n)$, respectively, such that $\left(x_{1}^{*}, x_{2}^{*}, \ldots, x_{n}^{*}\right) \in \Xi$.

Proof. As in the proof of Theorem 4.7, we know that there exists an unique point $\left(x_{1}^{*}, x_{2}^{*}, \ldots\right.$, $\left.x_{n}^{*}\right) \in E^{n}$ such that $\left(x_{1}^{*}, x_{2}^{*}, \ldots, x_{n}^{*}\right) \in \Xi$, and so

$$
\begin{gathered}
x_{1}^{*}=R_{\rho_{1}}^{M_{1}}\left(g_{1}\left(x_{2}^{*}\right)-\rho_{1} A_{1}\left(x_{2}^{*}, x_{1}^{*}\right)\right), \\
x_{2}^{*}=R_{\rho_{2}}^{M_{2}}\left(g_{2}\left(x_{3}^{*}\right)-\rho_{2} A_{2}\left(x_{3}^{*}, x_{2}^{*}\right)\right), \\
\vdots \\
x_{n-1}^{*}=R_{\rho_{n-1}}^{M_{n-1}}\left(g_{n-1}\left(x_{n}^{*}\right)-\rho_{n-1} A_{n-1}\left(x_{n}^{*}, x_{n-1}^{*}\right)\right), \\
x_{n}^{*}=R_{\rho_{n}}^{M_{n}}\left(g_{n}\left(x_{1}^{*}\right)-\rho_{n} A_{n}\left(x_{1}^{*}, x_{n}^{*}\right)\right),
\end{gathered}
$$

Note that

$$
\begin{aligned}
\left\|x_{1, k+1}-x_{1}^{*}\right\|= & \left\|\left(1-\alpha_{k}\right) x_{1, k}+\alpha_{k} R_{\rho_{1}}^{M_{1}}\left(g_{1}\left(x_{2, k+1}\right)-\rho_{1} A_{1}\left(x_{2, k+1}, x_{1, k}\right)\right)-x_{1}^{*}\right\| \\
= & \left\|\left(1-\alpha_{k}\right)\left(x_{1, k}-x_{1}^{*}\right)+\alpha_{k}\left[R_{\rho_{1}}^{M_{1}}\left(g_{1}\left(x_{2, k+1}\right)-\rho_{1} A_{1}\left(x_{2, k+1}, x_{1, k}\right)\right)-x_{1}^{*}\right]\right\| \\
= & \| \alpha_{k}\left[R_{\rho_{1}}^{M_{1}}\left(g_{1}\left(x_{2, k+1}\right)-\rho_{1} A_{1}\left(x_{2, k+1}, x_{1, k}\right)\right)-R_{\rho_{1}}^{M_{1}}\left(g_{1}\left(x_{2}^{*}\right)-\rho_{1} A_{1}\left(x_{2}^{*}, x_{1}^{*}\right)\right)\right] \\
& +\left(1-\alpha_{k}\right)\left(x_{1, k}-x_{1}^{*}\right) \| \\
\leq & \alpha_{k}\left\|R_{\rho_{1}}^{M_{1}}\left(g_{1}\left(x_{2, k+1}\right)-\rho_{1} A_{1}\left(x_{2, k+1}, x_{1, k}\right)\right)-R_{\rho_{1}}^{M_{1}}\left(g_{1}\left(x_{2}^{*}\right)-\rho_{1} A_{1}\left(x_{2}^{*}, x_{1}^{*}\right)\right)\right\| \\
& +\left(1-\alpha_{k}\right)\left\|x_{1, k}-x_{1}^{*}\right\| \\
\leq & \alpha_{k}\left\|\left(g_{1}\left(x_{2, k+1}\right)-\rho_{1} A_{1}\left(x_{2, k+1}, x_{1, k}\right)\right)-\left(g_{1}\left(x_{2}^{*}\right)-\rho_{1} A_{1}\left(x_{2}^{*}, x_{1}^{*}\right)\right)\right\| \\
& +\left(1-\alpha_{k}\right)\left\|x_{1, k}-x_{1}^{*}\right\| \\
= & \alpha_{k}\left\|\left(g_{1}\left(x_{2, k+1}\right)-g_{1}\left(x_{2}^{*}\right)\right)-\rho_{1}\left(A_{1}\left(x_{2, k+1}, x_{1, k}\right)-A_{1}\left(x_{2}^{*}, x_{1}^{*}\right)\right)\right\| \\
& +\left(1-\alpha_{k}\right)\left\|x_{1, k}-x_{1}^{*}\right\| \\
\leq & \left(1-\alpha_{k}\right)\left\|x_{1, k}-x_{1}^{*}\right\|+\alpha_{k}\left\|\left(x_{2, k+1}-x_{2}^{*}\right)-\left(g_{1}\left(x_{2, k}\right)-g_{1}\left(x_{2}^{*}\right)\right)\right\| \\
& +\alpha_{k}\left\|\left(x_{2, k+1}-x_{2}^{*}\right)-\rho_{1}\left(A_{1}\left(x_{2, k+1}, x_{1, k}\right)-A_{1}\left(x_{2}^{*}, x_{1}^{*}\right)\right)\right\| .
\end{aligned}
$$


Since $A_{j}: E \times E \rightarrow E$ are $\left(\mu_{j}, v_{j}\right)$-relaxed cocoercive and Lipschitz continuous in the first variable with constant $\tau_{j}$ and $g_{j}: E \rightarrow E$ are $\left(a_{j}, b_{j}\right)$-relaxed cocoercive and Lipschitz continuous with constant $\iota_{j}$ for $j \in\{1,2, \ldots, n\}$, we can conclude that

$$
\begin{aligned}
\widehat{L}_{A_{i}}^{*}= & \left\|x_{i+1, k+1}-x_{i+1}^{*}-\rho_{i}\left[A_{i}\left(x_{i+1, k+1}, x_{i, k}\right)-A_{i}\left(x_{i+1}^{*}, x_{i}^{*}\right)\right]\right\|^{q} \\
\leq & -q \rho_{i}\left\langle A_{i}\left(x_{i+1, k+1}, x_{i, k}\right)-A_{i}\left(x_{i+1}^{*}, x_{i}^{*}\right), J_{q}\left(x_{i+1, k+1}-x_{i+1}^{*}\right)\right\rangle \\
& +\left\|x_{i+1, k+1}-x_{i+1}^{*}\right\|^{q}+c_{q} \rho_{i}^{q}\left\|A_{i}\left(x_{i+1, k+1}, x_{i, k}\right)-A_{i}\left(x_{i+1}^{*}, x_{i}^{*}\right)\right\|^{q} \\
\leq & q \rho_{i}\left(\mu_{i}\left\|A_{i}\left(x_{i+1, k+1}, x_{i, k}\right)-A_{i}\left(x_{i+1}^{*}, x_{i}^{*}\right)\right\|^{q}-v_{i}\left\|x_{i+1, k+1}-x_{i+1}^{*}\right\|^{q}\right) \\
& +\left(1+c_{q} \rho_{i}^{q} \tau_{i}^{q}\right)\left\|x_{i+1, k+1}-x_{i+1}^{*}\right\|^{q} \\
\leq & \left(1+q \rho_{i} \mu_{i} \tau_{i}^{q}+c_{q} \rho_{i}^{q} \tau_{i}^{q}-q \rho_{i} v_{i}\right)\left\|x_{i+1, k+1}-x_{i+1}^{*}\right\|^{q}, \quad i=1,2, \ldots, n-1, \\
\widehat{L}_{g_{i}}^{*}= & \left\|x_{i+1, k+1}-x_{i+1}^{*}-\left(g_{i}\left(x_{i+1, k+1}\right)-g_{i}\left(x_{i+1}^{*}\right)\right)\right\|^{q} \\
\leq & -q\left\langle g_{i}\left(x_{i+1, k+1}\right)-g_{i}\left(x_{i+1}^{*}\right), J_{q}\left(x_{i+1, k+1}-x_{i+1}^{*}\right)\right\rangle \\
& +\left\|x_{i+1, k+1}-x_{i+1}^{*}\right\|^{q}+c_{q}\left\|g_{i}\left(x_{i+1, k+1}\right)-g_{i}\left(x_{i+1}^{*}\right)\right\|^{q} \\
\leq & \left(1+q a_{i} l_{i}^{q}+c_{q} l_{i}^{q}-q b_{i}\right)\left\|x_{i+1, k+1}-x_{i+1}^{*}\right\|^{q}, \quad i=1,2, \ldots, n-1, \\
\widehat{L}_{A_{n}}^{*}= & \left\|x_{1, k}-x_{1}^{*}-\rho_{n}\left[A_{n}\left(x_{1, k}, x_{n, k}\right)-A_{n}\left(x_{1}^{*}, x_{n}^{*}\right)\right]\right\|^{q} \\
\leq & \left\|x_{1, k}-x_{1}^{*}\right\|^{q}-q \rho_{n}\left\langle A_{n}\left(x_{1, k}, x_{n, k}\right)-A_{n}\left(x_{1}^{*}, x_{n}^{*}\right), J_{q}\left(x_{1, k}-x_{1}^{*}\right)\right\rangle \\
& +c_{q} \rho_{n}^{q}\left\|A_{n}\left(x_{1, k}, x_{n, k}\right)-A_{n}\left(x_{1}^{*}, x_{n}^{*}\right)\right\|^{q} \\
\leq & \left(1+q \rho_{n} \mu_{n} \tau_{n}^{q}+c_{q} \rho_{n}^{q} \tau_{n}^{q}-q \rho_{n} v_{n}\right)\left\|x_{1, k}-x_{1}^{*}\right\|^{q}, \\
\leq & \left(1+q a_{n} \iota_{n}^{q}+c_{q} q_{n}^{q}-q b_{n}\right)\left\|x_{1, k}-x_{1}^{*}\right\|^{q} . \\
\widehat{L}_{g_{n}}^{*}= & \left\|x_{1, k}-x_{1}^{*}-\left(g_{n}\left(x_{1, k}\right)-g_{n}\left(x_{1}^{*}\right)\right)\right\|^{q} \\
\leq & \left\|x_{1, k}-x_{1}^{*}\right\|^{q}-q\left\langle g_{n}\left(x_{1, k}\right)-g_{n}\left(x_{1}^{*}\right), J_{q}\left(x_{1, k}-x_{1}^{*}\right)\right\rangle+c_{q}\left\|g_{n}\left(x_{1, k}\right)-g_{n}\left(x_{1}^{*}\right)\right\|^{q} \\
\leq &
\end{aligned}
$$

Noticing that, for each $i \in\{1,2, \ldots, n-2\}$,

$$
\begin{gathered}
\left\|x_{i+1, k+1}-x_{i+1}^{*}\right\|=\| R_{\rho_{i+1}}^{M_{i+1}}\left(g_{i+1}\left(x_{i+2, k+1}\right)-\rho_{i+1} A_{i+1}\left(x_{i+2, k+1}, x_{i+1, k}\right)\right) \\
-R_{\rho_{i+1}}^{M_{i+1}}\left(g_{i+1}\left(x_{i+2}^{*}\right)-\rho_{i+1} A_{i+1}\left(x_{i+2}^{*}, x_{i+1}^{*}\right)\right) \| \\
\leq \|\left(g_{i+1}\left(x_{i+2, k+1}\right)-\rho_{i+1} A_{i+1}\left(x_{i+2, k+1}, x_{i+1, k}\right)\right) \\
\quad-\left(g_{i+1}\left(x_{i+2}^{*}\right)-\rho_{i+1} A_{i+1}\left(x_{i+2}^{*}, x_{i+1}^{*}\right)\right) \|
\end{gathered}
$$




$$
\begin{aligned}
= & \|\left(g_{i+1}\left(x_{i+2, k+1}\right)-g_{i+1}\left(x_{i+2}^{*}\right)\right)-\rho_{i+1} \\
& \times\left(A_{i+1}\left(x_{i+2, k+1}, x_{i+1, k}\right)-A_{i+1}\left(x_{i+2}^{*}, x_{i+1}^{*}\right)\right) \| \\
\leq & \left\|\left(x_{i+2, k+1}-x_{i+2}^{*}\right)-\rho_{i+1}\left(A_{i+1}\left(x_{i+2, k+1}, x_{i+1, k}\right)-A_{i+1}\left(x_{i+2}^{*}, x_{i+1}^{*}\right)\right)\right\| \\
& +\left\|\left(x_{i+2, k+1}-x_{i+2}^{*}\right)-\left(g_{i+1}\left(x_{i+2, k+1}\right)-g_{i+1}\left(x_{i+2}^{*}\right)\right)\right\| \\
\leq & \left(\sqrt[q]{1+q \rho_{i+1} \mu_{i+1} \tau_{i+1}^{q}+c_{q} \rho_{i+1}^{q} \tau_{i+1}^{q}-q \rho_{i+1} v_{i+1}}\right. \\
& \left.+\sqrt[q]{1+q a_{i+1} l_{i+1}^{q}+c_{q} q_{i+1}^{q}-q b_{i+1}}\right)\left\|x_{i+2, k+1}-x_{i+2}^{*}\right\|, \\
\left\|x_{n, k+1}-x_{n}^{*}\right\|= & \left\|R_{\rho_{n}}^{M_{n}}\left(g_{n}\left(x_{1, k}\right)-\rho_{n} A_{n}\left(x_{1, k}, x_{n, k}\right)\right)-R_{\rho_{n}}^{M_{n}}\left(g_{n}\left(x_{1}^{*}\right)-\rho_{n} A_{n}\left(x_{1}^{*}, x_{n}^{*}\right)\right)\right\| \\
\leq & \left\|\left(g_{n}\left(x_{1, k}\right)-\rho_{n} A_{n}\left(x_{1, k}, x_{n, k}\right)\right)-\left(g_{n}\left(x_{1}^{*}\right)-\rho_{n} A_{n}\left(x_{1}^{*}, x_{n}^{*}\right)\right)\right\| \\
= & \left\|\left(g_{n}\left(x_{1, k}\right)-g_{n}\left(x_{1}^{*}\right)\right)-\rho_{n}\left(A_{n}\left(x_{1, k}, x_{n, k}\right)-A_{n}\left(x_{1}^{*}, x_{n}^{*}\right)\right)\right\| \\
\leq & \left\|\left(x_{1, k}-x_{1}^{*}\right)-\left(\rho_{n} A_{n}\left(x_{1, k}, x_{n, k}\right)-\rho_{n} A_{n}\left(x_{1}^{*}, x_{n}^{*}\right)\right)\right\| \\
& +\left\|\left(x_{1, k}-x_{1}^{*}\right)-\left(g_{n}\left(x_{1, k}\right)-g_{n}\left(x_{1}^{*}\right)\right)\right\| \\
\leq & \left(\sqrt[q]{1+q \rho_{n} \mu_{n} \tau_{n}^{q}+c_{q} \rho_{n}^{q} \tau_{n}^{q}-q \rho_{n} v_{n}}+\sqrt[1]{1+q a_{n} q_{n}^{q}+c_{q} l_{n}^{q}-q b_{n}}\right) \\
& \times\left\|x_{1, k}-x_{1}^{*}\right\| .
\end{aligned}
$$

Consequently, we have

$$
\begin{aligned}
& \left\|x_{1, k+1}-x^{*}\right\| \\
& \leq \alpha_{k} \prod_{j=1}^{n}\left(\sqrt[q]{1+q \rho_{j} \mu_{j} \tau_{j}^{q}+c_{q} \rho_{j}^{q} \tau_{j}^{q}-q \rho_{j} v_{j}}+\sqrt[q]{1+q a_{j} \iota_{j}^{q}+c_{q} l_{j}^{q}-q b_{j}}\right)\left\|x_{1, k}-x_{1}^{*}\right\| \\
& +\left(1-\alpha_{k}\right)\left\|x_{1, k}-x^{*}\right\| \\
& =\left[1-\alpha_{k}\left(1-\prod_{j=1}^{n}\left(\sqrt[q]{1+q \rho_{j} \mu_{j} \tau_{j}^{q}+c_{q} \rho_{j}^{q} \tau_{j}^{q}-q \rho_{j} v_{j}}+\sqrt[q]{1+q a_{j} l_{j}^{q}+c_{q} l_{j}^{q}-q b_{j}}\right)\right)\right]\left\|x_{1, k}-x_{1}^{*}\right\| .
\end{aligned}
$$

Taking $\lambda_{k}=\alpha_{k}\left(1-\prod_{j=1}^{n}\left(\sqrt[q]{1+q \rho_{j} \mu_{j} \tau_{j}^{q}+c_{q} \rho_{j}^{q} \tau_{j}^{q}-q \rho_{j} v_{j}}+\sqrt[q]{1+q a_{j} \iota_{j}^{q}+c_{q} \iota_{j}^{q}-q b_{j}}\right)\right), C_{k}=D_{k}=0$, and $B_{k}=\left\|x_{1, k}-x_{1}^{*}\right\|$, then $B_{k+1} \leq\left(1-\lambda_{k}\right) B_{k}+C_{k}+D_{k}$. It follows from the conditions (i)-(iii) that

$$
\sum_{k=0}^{\infty} \lambda_{k}=\infty, \quad C_{k}=0\left(\lambda_{k}\right), \quad \sum_{k=0}^{\infty} D_{k}<\infty, \quad 0<\lambda_{k}<1, \forall k \in N
$$


Therefore, by Lemma 2.11 and (4.20), one has $\lim _{k \rightarrow \infty} B_{k}=0$. By the same argument of Theorem 4.7, we get

$$
\lim _{k \rightarrow \infty}\left\|x_{j, k}-x_{j}^{*}\right\|=0, \quad j=1,2, \ldots, n,
$$

that is, $x_{j, k} \rightarrow x_{j}^{*}$ as $k \rightarrow \infty$ for $j=1,2, \ldots, n$. Thus, $\left(x_{1, k}, x_{2, k}, \ldots, x_{n, k}\right)$ converges strongly to $\left(x_{1}^{*}, x_{2}^{*}, \ldots, x_{n}^{*}\right)$. This completes the proof.

By Remark 4.4, we have the following strong convergence theorems for the system of general variational inequalities problem (2.7).

Corollary 4.10. Let $K$ be a closed convex subset of a real Hilbert space $E$, and let $A_{j}$ and $g_{j}(j=1,2)$ be the same as in Theorem 3.4. Assume that $\left\{\alpha_{n}\right\}$ is a real sequence in $(0,1]$ and satisfies the following conditions:

(i) $\sum_{k=0}^{\infty} \alpha_{k}=\infty$;

(ii) $\sum_{k=0}^{\infty}\left\|e_{k}\right\|<+\infty$;

(iii) $\min \left\{1+2 a_{j} \iota_{j}^{2}+\iota_{j}^{2}-2 b_{j}, 1+2 \rho_{j} \mu_{j} \tau_{j}^{2}+\rho_{j}^{2} \tau_{j}^{2}-2 \rho_{j} v_{j}\right\} \geq 0, j=1,2$;

(iv) $0<\prod_{j=1}^{n}\left(\sqrt{1+2 a_{j} \iota_{j}^{2}+\iota_{j}^{2}-2 b_{j}}+\sqrt{1+2 \rho_{j} \mu_{j} \tau_{j}^{2}+\rho_{j}^{2} \tau_{j}^{2}-2 \rho_{j} v_{j}}\right)<1$.

Then the sequences $\left\{x_{j, k}\right\}(j=1,2)$ generated by Algorithm 4.5 converge strongly to $x_{j}^{*}(j=1,2)$, respectively, such that $\left(x_{1}^{*}, x_{2}^{*}\right)$ is the unique solution of the system of general variational inequalities problem (2.7).

Corollary 4.11. Let $K$ be a closed convex subset of a real Hilbert space $E$, and let $A_{j}$ and $g_{j}(j=1,2)$ be the same as in Theorem 3.4. Assume that $\left\{\alpha_{n}\right\}$ is a real sequence in $(0,1]$ and satisfies the following conditions:

(i) $\sum_{k=0}^{\infty} \alpha_{k}=\infty$;

(ii) $\min \left\{1+2 a_{j} \iota_{j}^{2}+\iota_{j}^{2}-2 b_{j}, 1+2 \rho_{j} \mu_{j} \tau_{j}^{2}+\rho_{j}^{2} \tau_{j}^{2}-2 \rho_{j} v_{j}\right\} \geq 0, j=1,2$;

(iii) $0<\prod_{j=1}^{n}\left(\sqrt{1+2 a_{j} \iota_{j}^{2}+\iota_{j}^{2}-2 b_{j}}+\sqrt{1+2 \rho_{j} \mu_{j} \tau_{j}^{2}+\rho_{j}^{2} \tau_{j}^{2}-2 \rho_{j} v_{j}}\right)<1$.

Then the sequences $\left\{x_{j, k}\right\}(j=1,2)$ generated by Algorithm 4.6 converge strongly to $x_{j}^{*}(j=1,2)$, respectively, such that $\left(x_{1}^{*}, x_{2}^{*}\right)$ is the unique solution of the system of general variational inequalities problem (2.7).

\section{An Application}

In this section, we applied the obtained results to study a class of bilevel variational inequalities in Hilbert space, which includes some bilevel programming as special cases and widely used in many practical problems. Moreover, an iterative algorithm and convergence theorem for solutions to the bilevel variational inequalities are given in Hilbert space.

Let $K_{1}$ and $K_{2}$ be nonempty closed convex subsets of a Hilbert space $E$, and let $g, h$ : $E \rightarrow E$ and $A: E \times E \rightarrow E$ be single-valued mappings. We consider the following bilevel variational inequalities (for short, (BVI)): find $\left(x^{*}, y^{*}\right) \in K_{1} \times K_{2}$ such that

$$
\left\langle x^{*}+h\left(y^{*}\right), h(x)-x^{*}\right\rangle \geq 0, \quad \forall x \in K_{1}, y^{*} \in \Psi\left(x^{*}\right),
$$


where $\Psi\left(x^{*}\right)$ is the solutions set of the following parametric variational inequalities with respect to the parametric variable $x^{*}$ :

$$
\left\langle\rho A\left(x^{*}, y^{*}\right)+y^{*}-g\left(x^{*}\right), g(y)-y^{*}\right\rangle \geq 0, \quad \forall y \in K_{2}
$$

where $\rho$ is a positive constant. Equations (5.1) and (5.2) are called the upper-level variation inequality (for short, (UVI)) and the lower-level variation inequality (for short, LVI), respectively. Denote the set of solutions to the (BVI) by $\Theta$. An important question for the (BVI) is how to solve the bilevel variational inequalities.

From Remark 2.9 and Theorem 3.1, one can easily conclude the following result.

Lemma 5.1. Let $\left(x^{*}, y^{*}\right) \in K_{1} \times K_{2}$. Then $\left(x^{*}, y^{*}\right)$ is a solution of the problem (BVI) if and only if $x^{*}=P_{K_{1}}\left(-h\left(y^{*}\right)\right)$, where $y^{*}=P_{K_{2}}\left(g\left(x^{*}\right)-\rho A\left(x^{*}, y^{*}\right)\right)$ and $\rho$ is a positive constant.

Lemma 5.2. Let $K_{1}$ and $K_{2}$ be nonempty closed convex subsets of a Hilbert space E. Let $A: E \times E \rightarrow$ $E$ be $(\mu, v)$-relaxed cocoercive and Lipschitz continuous in the first variable with constant $\tau$, and let $g: E \rightarrow E$ be $\left(a_{2}, b_{2}\right)$-relaxed cocoercive and Lipschitz continuous with constant $c_{2}$. Assume that $\left\{1+2 a_{2} c_{2}^{2}+c_{2}^{2}-2 b_{2}, 1+2 \rho \mu \tau^{2}+\rho^{2} \tau^{2}-2 \rho v\right\} \geq 0$ and

$$
0 \leq \sqrt{1+2 a_{2} c_{2}^{2}+c_{2}^{2}-2 b_{2}}+\sqrt{1+2 \rho \mu \tau^{2}+\rho^{2} \tau^{2}-2 \rho v}<1
$$

Then, for each $x \in K_{1}$, the parametric variational inequalities (5.2) have a uniquely solution. Further, the solution mapping $y(x)$ of the parametric variational inequalities (5.2) is continuous on $K_{1}$.

Proof. It directly follows from Theorems 3.2 and 3.4. This completes the proof.

Theorem 5.3. Let $K_{1}$ and $K_{2}$ be nonempty closed convex subsets of a Hilbert space E. Let $A: E \times E \rightarrow$ $E$ be $(\mu, v)$-relaxed cocoercive and Lipschitz continuous in the first variable with constant $\tau, h: E \rightarrow$ E $a\left(a_{1}, b_{1}\right)$-relaxed cocoercive and Lipschitz continuous with constant $c_{1}$, and let $g: E \rightarrow E$ be $a\left(a_{2}, b_{2}\right)$-relaxed cocoercive and Lipschitz continuous with constant $c_{2}$. Assume that $\left\{\alpha_{k}\right\}$ is a real sequence in $(0,1]$ and satisfies the following conditions:

(i) $\sum_{k=0}^{\infty} \alpha_{k}=\infty$;

(ii) $\min \left\{1+2 a_{j} c_{j}^{2}+c_{j}^{2}-2 b_{j}, 1+2 \rho \mu \tau^{2}+\rho^{2} \tau^{2}-2 \rho v, j=1,2\right\} \geq 0$;

(iii) $0<\prod_{j=1}^{n}\left(\sqrt[q]{1+q a_{j} l_{j}^{q}+c_{q} l_{j}^{q}-q b_{j}}+\sqrt[q]{1+q \rho_{j} \mu_{j} \tau_{j}^{q}+c_{q} \rho_{j}^{q} \tau_{j}^{q}-q \rho_{j} v_{j}}\right)<1$.

The sequences $\left\{x_{k}\right\}$ and $\left\{y_{k}\right\}$ generated by the following algorithm:

$$
\begin{gathered}
x_{0} \in K_{1}, \\
y_{k}=P_{K_{2}}\left(g\left(x_{k}\right)-\rho A\left(x_{k}, y_{k}\right)\right), \\
x_{k+1}=\left(1-\alpha_{k}\right) x_{k}+\alpha_{k} P_{K_{1}}\left(-h\left(y_{k}\right)\right), \quad k=0,1,2, \ldots,
\end{gathered}
$$

where $\rho$ is a positive constant. Then the sequences $\left\{x_{k}\right\}$ and $\left\{y_{k}\right\}$ converge strongly to $x^{*}$ and $y^{*}$, respectively, such that $\left(x^{*}, y^{*}\right)$ is a solution of the (BVI). 
Proof. The proof is similar to Remark 2.9 and Theorem 4.7, and so the proof is omitted. This completes the proof.

\section{Acknowledgments}

The authors would like to thank two anonymous referees for their valuable comments and suggestions, which led to an improved presentation of the results, and are grateful to Professor Yongkun $\mathrm{Li}$ as the editor of their paper. This work was supported by the Natural Science Foundation of China (nos. 71171150, 60804065), the academic award for excellent Ph.D. Candidates funded by Wuhan University and the Fundamental Research Fund for the Central Universities.

\section{References}

[1] G. Stampacchia, "Formes bilinéaires coercitives sur les ensembles convexes," Comptes-Rendus de l'Académie des Sciences de Paris, vol. 258, pp. 4413-4416, 1964.

[2] Q. H. Ansari, S. Schaible, and J. C. Yao, "System of vector equilibrium problems and its applications," Journal of Optimization Theory and Applications, vol. 107, no. 3, pp. 547-557, 2000.

[3] J.-S. Pang, "Asymmetric variational inequality problems over product sets: applications and iterative methods," Mathematical Programming, vol. 31, no. 2, pp. 206-219, 1985.

[4] X. Qin, S. Y. Cho, and S. M. Kang, "Convergence of an iterative algorithm for systems of variational inequalities and nonexpansive mappings with applications," Journal of Computational and Applied Mathematics, vol. 233, no. 2, pp. 231-240, 2009.

[5] R. U. Verma, "A-monotonicity and applications to nonlinear variational inclusion problems," Journal of Applied Mathematics and Stochastic Analysis, no. 2, pp. 193-195, 2004.

[6] R. P. Agarwal, N.-J. Huang, and M.-Y. Tan, "Sensitivity analysis for a new system of generalized nonlinear mixed quasi-variational inclusions," Applied Mathematics Letters, vol. 17, no. 3, pp. 345-352, 2004.

[7] J. W. Chen and Y. Z. Zou, "Existence of solutions of F-implicit variational inequality problems with extended projection operators," Acta Mathematica Sinica, vol. 53, no. 2, pp. 375-384, 2010.

[8] J. W. Peng, "A new system of generalized nonlinear mixed variational inclusions in Banach spaces," Fixed Point Theory and Applications, vol. 2010, Article ID 908490, 15 pages, 2010.

[9] N. J. Huang and Y. P. Fang, "Generalized m-accretive mappings in Banach spaces," Journal of Sichuan University, vol. 38, no. 4, pp. 591-592, 2001.

[10] N. J. Huang, "Random general set-valued strongly nonlinear quasivariational inequalities," Journal of Sichuan University, vol. 31, no. 4, pp. 420-425, 1994.

[11] N. J. Huang, "Generalized nonlinear variational inclusions with noncompact valued mappings," Applied Mathematics Letters, vol. 9, no. 3, pp. 25-29, 1996.

[12] N. J. Huang and Y. J. Cho, "Random completely generalized set-valued implicit quasi-variational inequalities," Positivity, vol. 3, no. 3, pp. 201-213, 1999.

[13] Y.-P. Fang, N. J. Huang, and H. B. Thompson, "A new system of variational inclusions with $\eta$ monotone operators in Hilbert spaces," Computers $\mathcal{E}$ Mathematics with Applications, vol. 49, no. 2-3, pp. 365-374, 2005.

[14] R. U. Verma, "Approximation-solvability of a class of A-monotone variational inclusion problems," Journal of the Korean Society Industrial and Applied Mathematics, vol. 8, no. 1, pp. 55-66, 2004.

[15] R. U. Verma, "Projection methods, algorithms, and a new system of nonlinear variational inequalities," Computers E Mathematics with Applications, vol. 41, no. 7-8, pp. 1025-1031, 2001.

[16] J. K. Kim and D. S. Kim, "A new system of generalized nonlinear mixed variational inequalities in Hilbert spaces," Journal of Convex Analysis, vol. 11, no. 1, pp. 235-243, 2004.

[17] Y. J. Cho, Y. P. Fang, N. J. Huang, and N. J. Hwang, "Algorithms for systems of nonlinear variational inequalities," Journal of the Korean Mathematical Society, vol. 41, no. 3, pp. 203-210, 2004.

[18] J. W. Peng and D. L. Zhu, "Existence of solutions and convergence of iterative algorithms for a system of generalized nonlinear mixed quasi-variational inclusions," Computers $\mathcal{E}$ Mathematics with Applications, vol. 53, no. 5, pp. 693-705, 2007. 
[19] X. Qin, S. S. Chang, Y. J. Cho, and S. M. Kang, "Approximation of solutions to a system of variational inclusions in Banach spaces," Journal of Inequalities and Applications, vol. 2010, Article ID 916806, 16 pages, 2010.

[20] U. Kamraksa and R. Wangkeeree, "A general iterative process for solving a system of variational inclusions in Banach spaces," Journal of Inequalities and Applications, vol. 2010, Article ID 190126, 24 pages, 2010.

[21] R. Wangkeeree and U. Kamraksa, "An iterative approximation method for solving a general system of variational inequality problems and mixed equilibrium problems," Nonlinear Analysis. Hybrid Systems, vol. 3, no. 4, pp. 615-630, 2009.

[22] N. Petrot, "A resolvent operator technique for approximate solving of generalized system mixed variational inequality and fixed point problems," Applied Mathematics Letters, vol. 23, no. 4, pp. 440445, 2010.

[23] Y. Zhao, Z. Xia, L. Pang, and L. Zhang, "Existence of solutions and algorithm for a system of variational inequalities," Fixed Point Theory and Applications, vol. 2010, Article ID 182539, 11 pages, 2010.

[24] J. W. Chen and Z. Wan, "Existence of solutions and convergence analysis for a system of quasivariational inclusions in Banach spaces," Journal of Inequalities and Applications. In press.

[25] Y.-C. Liou and J.-C. Yao, "Bilevel decision via variational inequalities," Computers E Mathematics with Applications, vol. 49, no. 7-8, pp. 1243-1253, 2005.

[26] L. J. Lin and H. J. Shie, "Existence theorems of quasivariational inclusion problems with applications to bilevel problems and mathematical programs with equilibrium constraint," Journal of Optimization Theory and Applications, vol. 138, no. 3, pp. 445-457, 2008.

[27] M. A. Noor and K. I. Noor, "Projection algorithms for solving a system of general variational inequalities," Nonlinear Analysis. Theory, Methods \& Applications, vol. 70, no. 7, pp. 2700-2706, 2009.

[28] R. U. Verma, "General over-relaxed proximal point algorithm involving A-maximal relaxed monotone mappings with applications," Nonlinear Analysis. Theory, Methods E Applications, vol. 71, no. 12, pp. e1461-e1472, 2009.

[29] Z. He and F. Gu, "Generalized system for relaxed cocoercive mixed variational inequalities in Hilbert spaces," Applied Mathematics and Computation, vol. 214, no. 1, pp. 26-30, 2009.

[30] S. S. Chang, H. W. Joseph Lee, and C. K. Chan, "Generalized system for relaxed cocoercive variational inequalities in Hilbert spaces," Applied Mathematics Letters, vol. 20, no. 3, pp. 329-334, 2007.

[31] R. U. Verma, "Generalized system for relaxed cocoercive variational inequalities and projection methods," Journal of Optimization Theory and Applications, vol. 121, no. 1, pp. 203-210, 2004.

[32] K. Aoyama, Y. Kimura, W. Takahashi, and M. Toyoda, "On a strongly nonexpansive sequence in Hilbert spaces," Journal of Nonlinear and Convex Analysis, vol. 8, no. 3, pp. 471-489, 2007.

[33] L. S. Liu, "Ishikawa and Mann iterative process with errors for nonlinear strongly accretive mappings in Banach spaces," Journal of Mathematical Analysis and Applications, vol. 194, no. 1, pp. 114-125, 1995.

[34] H. K. Xu, "Inequalities in Banach spaces with applications," Nonlinear Analysis. Theory, Methods $\mathcal{E}$ Applications, vol. 16, no. 12, pp. 1127-1138, 1991. 


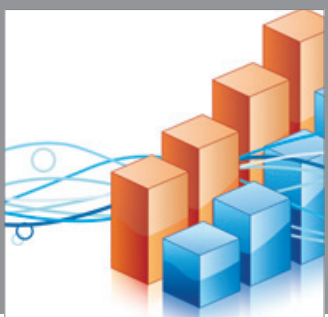

Advances in

Operations Research

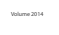

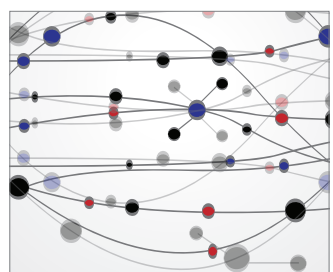

\section{The Scientific} World Journal
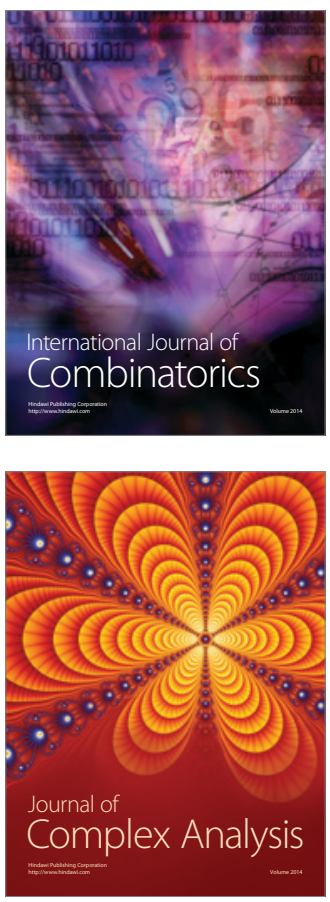

International Journal of

Mathematics and

Mathematical

Sciences
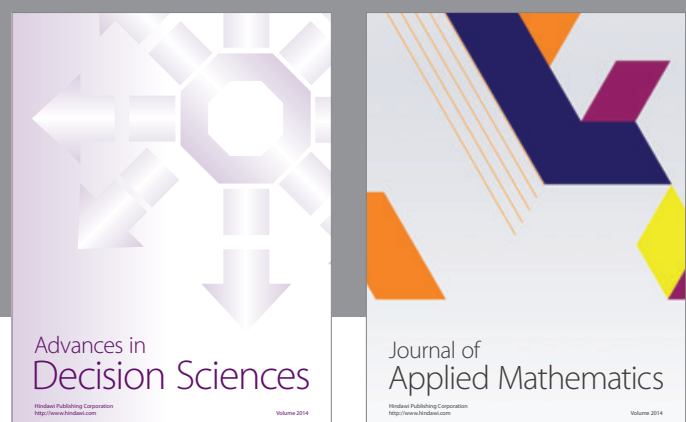

Journal of

Applied Mathematics
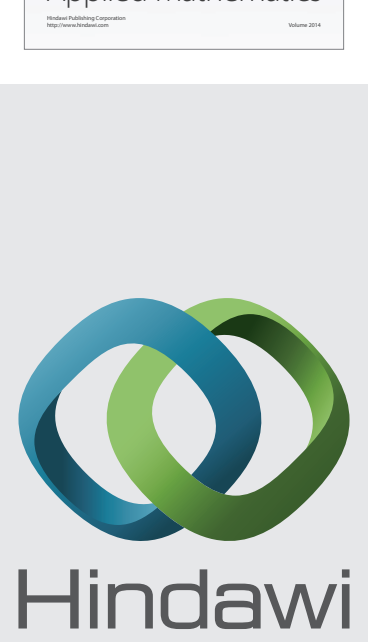

Submit your manuscripts at http://www.hindawi.com
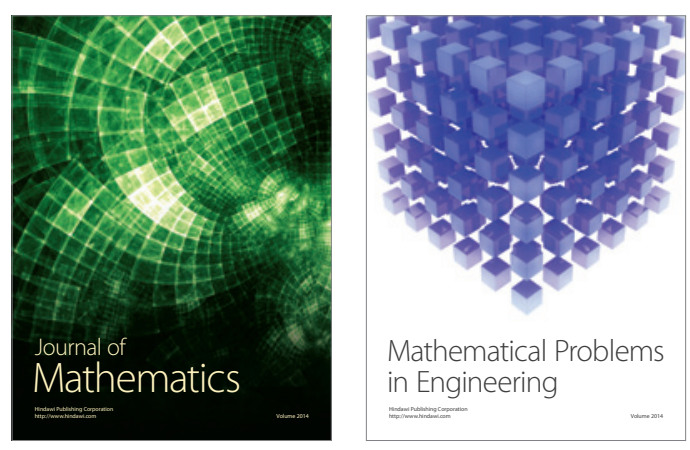

Mathematical Problems in Engineering
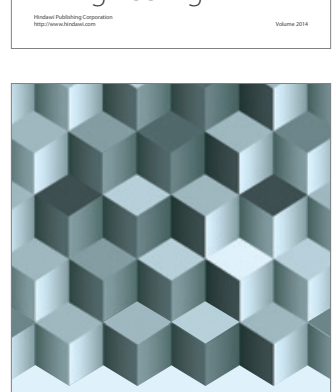

Journal of

Function Spaces
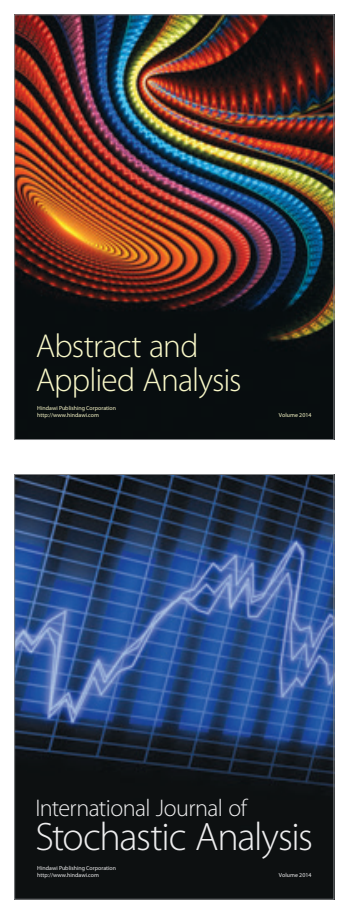

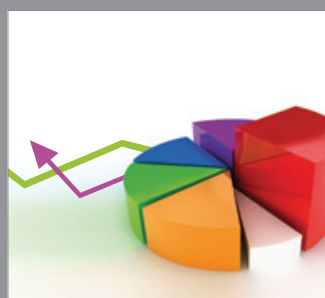

ournal of

Probability and Statistics

Promensencen
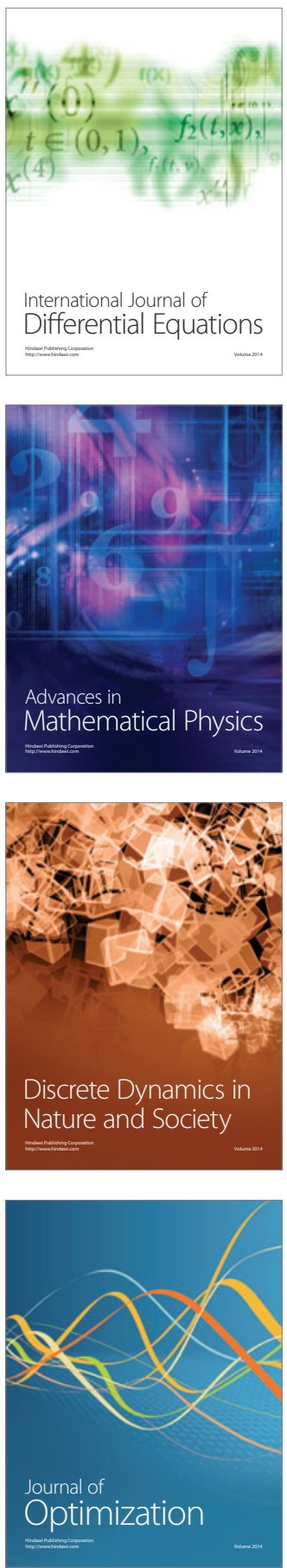